\title{
An angiogenesis model with nonlinear chemotactic response and flux at the tumor boundary
}

\author{
M. Delgado, I. Gayte, C. Morales-Rodrigo and A. Suárez, \\ Dpto. de Ecuaciones Diferenciales y Análisis Numérico \\ Fac. de Matemáticas, Univ. de Sevilla \\ C/. Tarfia s/n, 41012 - Sevilla, SPAIN, \\ E-mail addresses: madelgado@us.es, gayte@us.es, cristianmatematicas@yahoo.com, \\ suarez@us.es
}

\begin{abstract}
The main goal of this paper is the theoretical study of a system modelling the angiogenesis process. This model presents ...
\end{abstract}

Key Words. Angiogenesis, chemotaxis, bifurcation methods, coexistence state.

\section{Introduction}

In this paper we analyze a system modelling a crucial step in the tumor growth process: the angiogenesis. We suggest to the interested reader the paper [15] to know multiple aspects of angiogenesis. We focus our attention only on the behaviour of two populations involved in such process: the endothelial cells (CEs) which move and reproduce to generate a new vascular net attracted by the chemical substance generated by the tumor (TAF). We represent them by $u$ and $v$ respectively. They live together in a region $\Omega \subset \mathbb{R}^{N}$, $N \geq 1$, that is assumed to be bounded and connected and with a regular boundary $\partial \Omega$. Specifically, we consider the case in which

$$
\partial \Omega=\Gamma_{1} \cup \Gamma_{2},
$$

with $\Gamma_{1} \cap \Gamma_{2}=\emptyset$, being $\Gamma_{i}$ closed and open in the relative topology of $\partial \Omega$. We assume that $\Gamma_{2}$ is the boundary of the tumor and $\Gamma_{1}$ is the boundary of the blood vessel, see Figure 1 where we have represented a particular situation, in this case the tumor is surrounded by the vessel.

We assume Neumann homogeneous boundary conditions in both variables at $\Gamma_{1}$, and also for the variable $u$ at $\Gamma_{2}$. However, and as one of the principal novelty of this model, we consider that at the boundary of the tumor, this generates a quantity of TAF depending nonlinearly of the TAF existing. Specifically, we assume that at $\Gamma_{2}$

$$
\frac{\partial v}{\partial n}=\mu \frac{v}{1+v}
$$




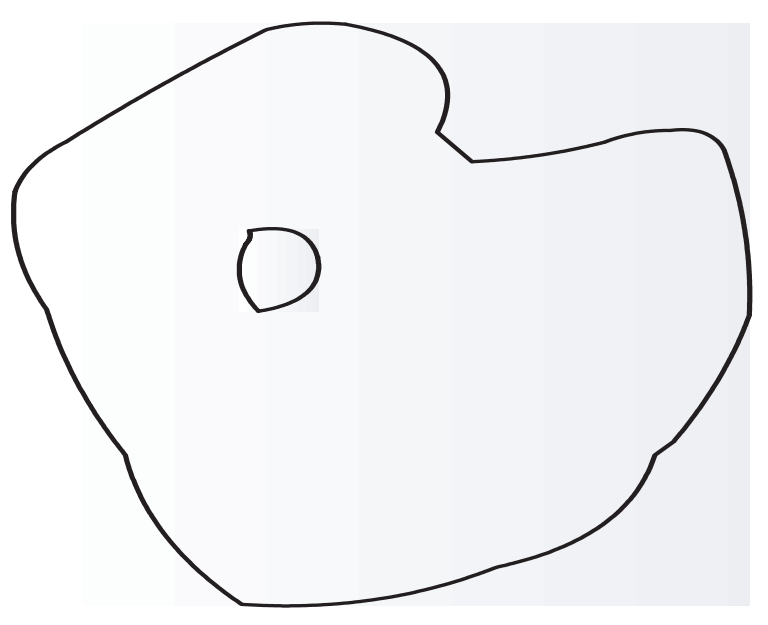

Figure 1: A particular example of domain $\Omega$.

being $\mu$ a real number, although in the real application $\mu$ will be a positive constant. In such case, $\mu$ represents the rate of TAF produced. Here $n$ stands for the normal outward vector to $\partial \Omega$.

So, we are assuming that the tumor is generating the TAF with a production term of the Michaelis-Menten type, in contrast to the model in [7] where this term is linear.

Hence, we study the following parabolic problem and its stationary counterpart

$$
\begin{cases}u_{t}-\Delta u=-\operatorname{div}(V(u) \nabla v)+\lambda u-u^{2} & \text { in } \Omega \times(0, T), \\ v_{t}-\Delta v=-v-c u v & \text { in } \Omega \times(0, T), \\ \frac{\partial u}{\partial n}=\frac{\partial v}{\partial n}=0 & \text { on } \Gamma_{1} \times(0, T), \\ \frac{\partial u}{\partial n}=0, \quad \frac{\partial v}{\partial n}=\mu \frac{v}{1+v} & \text { on } \Gamma_{2} \times(0, T), \\ u(x, 0)=u_{0}(x), \quad v(x, 0)=v_{0}(x) & \text { in } \Omega,\end{cases}
$$

where $0<T \leq+\infty, \lambda, \mu \in \mathbb{R}, c>0$ and $V \in C^{1}(\mathbb{R}), V>0$ in $(0, \infty)$ with $V(0)=0$; and $u_{0}$ and $v_{0}$ are non-negative and non-trivial given functions.

Let us explain now the model. We are assuming that $u$ is affected by a chemotaxis term. Here, $V$ models the chemotactic response of the CEs to the chemoatractant TAF, and in this case this response depends on the density of $u$ on a non-linear way. Also, we assume that CEs grow following a logistic law. On the other hand, we assume that the TAF has a degradation typically linear, $-v$, it is also affected by a competition term with $u,-c u v$.

Similar models to (1.1) have been studied extensively in the last years, we refer to the recent review paper [11].

Model (1.1) has basically three main difficulties, due basically to the nonlinearities: the reaction term, the chemotactic response and the boundary condition. The logistic term has been yet used to model the cell growth and death. Also, the nonlinear chemotactic sensitivity has been used in different papers, see for instance [12], [16], [13] and references 
therein. We would like to mention that in [12] the function $V$ is bounded and negative for large values of $u$, which provides bounds of the solution and so prevents the overcrowding.

However, the non-linear term at the boundary of the tumor has not used extensively in our knowledge. The combination of these three terms imply a more involved and realistic model.

We summarize our main results. With respect to the parabolic problem, and using basically [3] and some estimates:

- There exists a unique local in time positive solution of (1.1).

- If $V$ is bounded, there exists a unique global in time positive solution of (1.1).

With respect to the stationary problem, it is clear that there exists three kinds of solutions of (1.1): the trivial one, the semi-trivial solutions $(u, 0)$ and $(0, v)$ and the solutions with both components positive, the coexistence states $(u, v)$. Basically, the trivial solution always exists, and:

- The semi-trivial solution $(u, 0)$ exists if, and only if $\lambda>0$. In fact this semi-trivial solution is $(\lambda, 0)$.

- There exists a value $\mu_{1}>0$ such that the semi-trivial solution $(0, v)$ exists if, and only if $\mu>\mu_{1}$.

With respect to the existence of coexistence states, we need to introduce two functions $F:(0,+\infty) \mapsto \mathbb{R}$ and $\Lambda:\left(\mu_{1},+\infty\right) \mapsto \mathbb{R}$ such that:

- If $\lambda \leq 0$ or $\mu \leq \mu_{1}$ there does not exist any coexistence state of (3.1).

- Assume that $V^{\prime}(0)>0$, there exists at least a coexistence state of (3.1) if

$$
(\mu-F(\lambda))(\lambda-\Lambda(\mu))>0 .
$$

- Assume that $V^{\prime}(0)=0$, there exists at least a coexistence state of (3.1) if $\lambda>0$ and

$$
\mu-F(\lambda)>0 .
$$

Finally, with respect to the stability of the semi-trivial solutions, we show that

- $(u, 0)$ is stable if $\mu<F(\lambda)$, and unstable if $\mu>F(\lambda)$.

- $(0, v)$ is stable if $\lambda<\Lambda(\mu)$ (resp. $\lambda<0$ if $V^{\prime}(0)=0$ ), and unstable if $\lambda>\Lambda(\mu)$ (resp. $\lambda>0$ if $\left.V^{\prime}(0)=0\right)$.

So, when both semi-trivial solutions are stable or unstable, there exists at least one coexistence state. Hence, these curves are crucial in the study of existence of positive solutions and we will study in detail both maps.

In order to prove these results we use mainly bifurcation methods and sub and supersolution.

The structure of this paper is as follows. In section 2 we study the parabolic problem: first we prove the existence of solution local in time and then the global existence. In section 3 we study the stationary problem. Section 4 is devoted to study the global stability of the semi-trivial solution $(u, 0)$. Finally in the last section we briefly discuss some biological implications of our results. 


\section{The parabolic problem}

\section{$2.1 \quad$ Local existence}

We are interested by the positive solution of the following system of PDEs

$$
\begin{cases}u_{t}-\Delta u=-\operatorname{div}(V(u) \nabla v)+\lambda u-u^{2} & \text { in } \Omega \times(0, T), \\ v_{t}-\Delta v=-v-c u v & \text { in } \Omega \times(0, T), \\ \frac{\partial u}{\partial n}=\frac{\partial v}{\partial n}=0 & \text { on } \Gamma_{1} \times(0, T), \\ \frac{\partial u}{\partial n}=\frac{\partial v}{\partial n}=\mu \frac{v}{1+v} & \text { on } \Gamma_{2} \times(0, T), \\ u(x, 0)=u_{0}(x), & \text { in } \Omega, \\ v(x, 0)=v_{0}(x), & \text { in } \Omega,\end{cases}
$$

where $c$ is a positive constant and $\lambda \in \mathbb{R}$. The following result shows the local existence of solution of (2.1).

Theorem 2.1. Let $p>N$ and suppose that the initial data $\left(u_{0}, v_{0}\right) \in W^{1, p}\left(\Omega ; \mathbb{R}^{2}\right)$ and $u_{0} \geq 0, v_{0} \geq 0$ a.e. Then, problem (2.1) has a unique nonnegative local in time classical solution

$$
(u, v) \in C\left(\bar{\Omega} \times\left(0, T_{\max }\right) ; \mathbb{R}^{2}\right) \cap C^{2,1}\left(\bar{\Omega} \times\left(0, T_{\max }\right) ; \mathbb{R}^{2}\right),
$$

where $T_{\max }$ denotes the maximal existence time. Moreover, if there exists a function $w:(0,+\infty) \rightarrow(0,+\infty)$ such that, for each $T>0$,

$$
\|(u(t), v(t))\|_{\infty} \leq w(T), \quad 0<t<\min \left\{T, T_{\max }\right\},
$$

then $T_{\max }=+\infty$.

Proof. We will prove that problem (2.1) is included in the frame of [3]. Let $\delta>0$ be and denote $D_{0}=(-\delta, \infty) \times(-\delta,+\infty)$ which is an open set containing the range of the solutions $u$ and $v$. For $n=2$, number of equations, we define $n^{2}$ functions $a_{j k} \in C^{2}\left(D_{0} ; \mathcal{L}\left(\mathbb{R}^{2}\right)\right)$ for $1 \leq j, k \leq n$ in the following way. For each $\left(\eta_{1}, \eta_{2}\right) \in D_{0}$

$$
a_{j k}\left(\begin{array}{c}
\eta_{1} \\
\eta_{2}
\end{array}\right)=\left(\begin{array}{cc}
0 & 0 \\
0 & 0
\end{array}\right) \quad \text { if } j \neq k ; \quad a_{j k}\left(\begin{array}{c}
\eta_{1} \\
\eta_{2}
\end{array}\right)=\left(\begin{array}{cc}
1 & 0 \\
-V\left(\eta_{2}\right) & 1
\end{array}\right) \quad \text { if } j=k
$$

then, we put

$$
\mathcal{A}\left(\begin{array}{l}
v \\
u
\end{array}\right)\left(\begin{array}{l}
v \\
u
\end{array}\right):=-\sum_{j, k=1}^{N} \partial_{j}\left(a_{j k}\left(\begin{array}{l}
v \\
u
\end{array}\right)\left(\begin{array}{c}
\partial_{k} v \\
\partial_{k} u
\end{array}\right)\right)=-\left(\begin{array}{c}
\operatorname{div}(\nabla v) \\
\operatorname{div}(\nabla u-V(u) \nabla v)
\end{array}\right) .
$$

For the boundary conditions, we define for $i=1,2, \delta_{i}: \partial \Omega \rightarrow\{0,1\}$ as $\delta_{i}(x)=1 \forall x \in$ $\Gamma_{1} \cup \Gamma_{2}$, because the boundary conditions are Neumann boundary conditions for each unknown on each part of the boundary. We denote $\left(c_{i j}\right) 1 \leq i, j \leq 2$ the following matrix:

$$
c_{11}\left(\begin{array}{l}
\eta_{1} \\
\eta_{2}
\end{array}\right)=\left\{\begin{array}{cc}
0 & \text { on } \Gamma_{1} \\
-\frac{\mu}{1+\eta_{2}} & \text { on } \Gamma_{2}
\end{array} \quad c_{12}\left(\begin{array}{l}
\eta_{1} \\
\eta_{2}
\end{array}\right)= \begin{cases}0 & \text { on } \Gamma_{1} \\
0 & \text { on } \Gamma_{2}\end{cases}\right.
$$




$$
c_{21}\left(\begin{array}{c}
\eta_{1} \\
\eta_{2}
\end{array}\right)=\left\{\begin{array}{cc}
0 & \text { on } \Gamma_{1} \\
\frac{\mu V\left(\eta_{2}\right)}{1+\eta_{1}} & \text { on } \Gamma_{2}
\end{array} c_{22}\left(\begin{array}{c}
\eta_{1} \\
\eta_{2}
\end{array}\right)=\left\{\begin{array}{cc}
0 & \text { on } \Gamma_{1} \\
0 & \text { on } \Gamma_{2}
\end{array},\right.\right.
$$

and

$$
\delta:=\operatorname{diag}\left(\delta_{i}\right): \partial \Omega \longrightarrow \mathcal{L}\left(\mathbb{R}^{2}\right) .
$$

Then we put

$$
\begin{gathered}
\mathcal{B}\left(\begin{array}{l}
v \\
u
\end{array}\right)\left(\begin{array}{l}
v \\
u
\end{array}\right):=\left(\begin{array}{cc}
\delta_{1} & 0 \\
0 & \delta_{2}
\end{array}\right)\left[\sum_{j=1}^{N} n_{j} \gamma\left(a_{j k}\left(\begin{array}{l}
v \\
u
\end{array}\right)\left(\begin{array}{l}
\partial_{k} v \\
\partial_{k} u
\end{array}\right)\right)+\right. \\
\gamma\left(\left(\begin{array}{ll}
c_{11} & c_{12} \\
c_{21} & c_{22}
\end{array}\right)\left(\begin{array}{l}
v \\
u
\end{array}\right)\right)+\left(\begin{array}{cc}
1-\delta_{1} & 0 \\
0 & 1-\delta_{2}
\end{array}\right) \gamma\left(\begin{array}{l}
v \\
u
\end{array}\right)= \\
\left(\begin{array}{c}
\frac{\partial v}{\partial n}-\frac{\mu v}{1+v} \\
-V(u) \frac{\partial v}{\partial n}+\frac{\partial u}{\partial n}+\frac{\mu V(u) v}{1+v}
\end{array}\right)
\end{gathered}
$$

This couple $(\mathcal{A}, \mathcal{B})$ is a linear boundary value problem of separated divergence form, namely, if we denote

$$
\alpha=I_{2}, \quad \text { and } \quad a=\left(\begin{array}{cc}
1 & 0 \\
-V(u) & 1
\end{array}\right)
$$

it holds that

$$
a_{j k}=\alpha_{j k} a
$$

where $\alpha$ is symmetric and uniformly positive definite and $(\mathcal{A}, \mathcal{B})$ is normally elliptic because it also holds

$$
\sigma(a(x)) \subset[\operatorname{Re} z>0] \quad \forall x \in \bar{\Omega}
$$

and the boundary operator is a Neumann boundary operator of each component of $\partial \Omega$. Then, if we denote

$$
W_{\mathcal{B}}^{2, p}:=\operatorname{Ker} \mathcal{B}=\left\{(v, u) \in\left(W^{2, p}(\Omega)\right)^{2}: \mathcal{B}(v, u)^{t}=0\right\}
$$

and $L^{p}=\left(L^{p}(\Omega)\right)^{2}$, then the operator

$$
A:=\left.\mathcal{A}\right|_{W_{\mathcal{B}}^{2, p}} \in \mathcal{L}\left(W_{\mathcal{B}}^{2, p}, L^{p}\right)
$$

is well defined and is the negative infinitesimal generator of an analytic semigroup on $L^{p}$ (pg 19).

For the reaction term, we define the function $f \in C^{2}\left(D_{0} ; \mathbb{R}^{2}\right)$ by

$$
f\left(\begin{array}{l}
\eta_{1} \\
\eta_{2}
\end{array}\right)=\left(\begin{array}{l}
-\eta_{1}-c \eta_{1} \eta_{2} \\
\lambda \eta_{2}-\eta_{2}^{2}
\end{array}\right)
$$


Then, (2.1) can be written as the following quasilinear parabolic boundary value problem

$$
\begin{aligned}
& \partial_{t}\left(\begin{array}{l}
v \\
u
\end{array}\right)+\mathcal{A}\left(\begin{array}{l}
v \\
u
\end{array}\right)\left(\begin{array}{l}
v \\
u
\end{array}\right)=f\left(\begin{array}{l}
v \\
u
\end{array}\right) \text { in } \Omega \times(0, \infty) \\
& \mathcal{B}\left(\begin{array}{l}
v \\
u
\end{array}\right)\left(\begin{array}{l}
v \\
u
\end{array}\right)=\left(\begin{array}{l}
0 \\
0
\end{array}\right) \quad \text { on } \partial \Omega \times(0, \infty) \\
& \left(\begin{array}{l}
v(x, 0) \\
u(x, 0)
\end{array}\right)=\left(\begin{array}{l}
v_{0}(x) \\
u_{0}(x)
\end{array}\right) \quad \text { on } \Omega
\end{aligned}
$$

Then, Theorems 14.4 and 14.6 of [3] are applicable. The first one says that with our hypotheses ( $p>N$, the coefficients of $\mathcal{A}$ and $\mathcal{B}$ are $C^{2}, f \in C^{2}$ and independent of the gradient) for each initial data belonging to $W_{\mathcal{B}}^{s, p}$, there exists a unique maximal weak

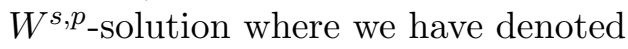

$$
W_{\mathcal{B}}^{s, p}= \begin{cases}\left\{(v, u) \in W^{s, p}(\Omega)^{2}: \mathcal{B}(v, u)^{t}=0\right\} & \text { if } 1+\frac{1}{p}<s \leq 2 \\ W^{s, p} & \text { if } 0 \leq s<1+\frac{1}{p}\end{cases}
$$

This function is a $W^{1, q}$-solution for each $q \in(1, \infty)$ (Coroll. 14.5). In particular, for $s=1$, our claim follows. The second one asserts that because the boundary operator is equal to 0 , the solution is a classical solution and the equation is verified point-wise.

The nonnegativity of the solution follows from Theorem 15.1. In fact, the hypothesis (15.3) is verified for $r=2$ because $V(0)=0$ and, so, the nonnegativity of $u$ holds. But, if $u \geq 0$, then the maximum principle applied to the problem

$$
\begin{cases}v_{t}-\Delta v=-v-c u v & \text { in } \Omega \times(0, T), \\ \frac{\partial v}{\partial n}=0 & \text { on } \Gamma_{1} \times(0, T), \\ \frac{\partial v}{\partial n}=\mu \frac{v}{1+v} & \text { on } \Gamma_{2} \times(0, T), \\ v(x, 0)=v_{0}(x) & \text { in } \Omega,\end{cases}
$$

implies that $v \geq 0$.

To reach the result about the global solution, we can invoke Theorem 15.5. which is applicable because $(\mathcal{A}, \mathcal{B})$ is a lower triangular system. For these systems and if $a_{j k}$ is a diagonal matrix, $f$ is independent of the gradient and if there exists a function $w: \mathbb{R}_{+} \rightarrow$ $\mathbb{R}_{+}$such that

$$
\|\left(u(t), v(t) \|_{\infty} \leq w(T), \quad 0 \leq t \leq T<\infty, t<t^{+},\right.
$$

then $t^{+}=\infty$, supposed that the solution is bounded away from $\partial \Omega$ for each $T>0$.

\subsection{Global existence}

The following lemma states that $v$ is bounded, independently of the variables $t$ and $x$, via the well-known method of sub and supersolutions (see for example [17]). 
Lemma 2.2. There exists a constant $C>0$ such that the $v$-solutions of (1.1) satisfy

$$
\|v(t)\|_{C(\bar{\Omega})} \leq C
$$

where $C$ is independent of $t$.

Proof. Observe that the $v$-solutions of the problem (1.1) are subsolutions of

$$
\left\{\begin{array}{lll}
w_{t}-\Delta w=-w & \text { in } & \Omega \times\left(0, T_{\max }\right), \\
\frac{\partial w}{\partial n}=0 & \text { on } & \Gamma_{1} \times\left(0, T_{\max }\right), \\
\frac{\partial w}{\partial n}=|\mu| & \text { on } & \Gamma_{2} \times\left(0, T_{\max }\right), \\
v(x, 0)=v_{0}(x) & \text { in } & \Omega .
\end{array}\right.
$$

Now, let $\varphi$ be the solution of the stationary problem

$$
\left\{\begin{array}{l}
-\Delta \varphi+\varphi=0 \text { in } \Omega \\
\partial_{n} \varphi=0 \text { on } \Gamma_{1}, \partial_{n} \varphi=|\mu| \text { on } \Gamma_{2} .
\end{array}\right.
$$

It is well known that $\varphi>0$. Taking $K>0$ big enough, $K \varphi$ is a supersolution of (2.5). Therefore, $v \leq w \leq K \varphi$ and the conclusion is easily followed.

Lemma 2.3. There exists $C>0$ such that the solution of the u-equation of (1.1) satisfies

$$
\|u(t)\|_{L^{2}(\Omega)} \leq C
$$

with a constant $C$ which is independent of $t$.

Proof. Multiplying the $v$-equation by $v$ and integrating in $\Omega$, we obtain

$$
\frac{d}{2 d t} \int_{\Omega} v^{2}+\int_{\Omega} v^{2}+\int_{\Omega}|\nabla v|^{2}=\int_{\partial \Omega} \frac{\mu v}{1+v}-\int_{\Omega} c u v^{2}
$$

Adding the term $-\frac{C \varepsilon}{2} \int_{\Omega} v^{2}$ on both sides of the equality, where $C$ is the constant of the injection of $H^{1}(\Omega)$ in $L^{2}\left(\Gamma_{2}\right)$, taking into account Lemma 2.2 and multiplying the before equality by $e^{(2-C \varepsilon) t}$, we have

$$
\frac{d}{d t}\left(e^{(2-C \varepsilon) t} \int_{\Omega} v^{2}\right)+2 e^{(2-C \varepsilon) t} \int_{\Omega}|\nabla v|^{2} \leq 2|\mu| C|\partial \Omega| e^{(2-C \varepsilon) t},
$$

where $|\partial \Omega|$ denotes the $N-1$ dimensional Lebesgue measure of $\partial \Omega$. Therefore, integrating in $(0, t)$ and multiplying by $e^{-(2-C \varepsilon) t}$ we get

$$
\begin{gathered}
\int_{\Omega} v^{2}(t)+e^{-(2-C \varepsilon) t} 2 \int_{0}^{t} e^{(2-C \varepsilon) s}\left(\int_{\Omega}|\nabla v|^{2}\right) d s \leq \\
\left(2|\mu| C|\partial \Omega| \int_{0}^{t} e^{(2-C \varepsilon) s} d s\right) e^{-(2-C \varepsilon) t}+\int_{\Omega} v_{0}^{2} e^{-(2-C \varepsilon) t} .
\end{gathered}
$$


In particular, from (2.6) we obtain

$$
e^{-(2-C \varepsilon) t} \int_{0}^{t} e^{(2-C \varepsilon) s}\left(\int_{\Omega}|\nabla v|^{2}\right) d s \leq C\left(\left\|v_{0}\right\|_{L^{2}(\Omega)}\right)
$$

Next, we multiply the $u$-equation by $u$ and integrate in $\Omega$

$$
\frac{d}{d t} \int_{\Omega} u^{2}=-2 \int_{\Omega}|\nabla u|^{2}+2 \int_{\Omega} V(u) \nabla u \cdot \nabla v-2 \int_{\partial \Omega} V(u) \frac{\mu u v}{1+v}+\int_{\Omega} 2 \lambda u^{2}-2 \int_{\Omega} u^{3} .
$$

Adding $2 \int_{\Omega} u^{2}$ on both sides of the equality we have

$\frac{d}{d t} \int_{\Omega} u^{2}+2 \int_{\Omega} u^{2}=-2 \int_{\Omega}|\nabla u|^{2}+2 \int_{\Omega} V(u) \nabla u \cdot \nabla v-2 \int_{\Gamma_{2}} V(u) \frac{\mu u v}{1+v}+2 \int_{\Omega}(\lambda+1) u^{2}-u^{3}$.

Owing to $|V(s)| \leq C$ for almost every $s \in \mathbf{R}$ and the inequality $(\lambda+1) s^{2}-s^{3} \leq C=C(\lambda)$ for all $s \geq 0$, we deduce

$$
\frac{d}{d t} \int_{\Omega} u^{2}+2 \int_{\Omega} u^{2} \leq-2 \int_{\Omega}|\nabla u|^{2}+C \int_{\Omega} 2|\nabla u||\nabla v|+C \int_{\Gamma_{2}}|\mu| \frac{u v}{1+v}+2 C|\Omega| .
$$

The term $C|\mu| \int_{\Gamma_{2}} \frac{u v}{1+v}$ is bounded by the following expressions

$$
C|\mu| \int_{\Gamma_{2}} \frac{u v}{1+v} \leq C|\mu| \int_{\Gamma_{2}} u \leq \varepsilon \int_{\Gamma_{2}} u^{2}+C(\varepsilon),
$$

using that for every $\varepsilon>0$ there exists $C(\varepsilon)>0$ such that $s \leq \varepsilon s^{2}+C(\varepsilon)$ for every $s \in \mathbf{R}$. Moreover,

$$
\varepsilon \int_{\Gamma_{2}} u^{2} \leq C\left(\varepsilon \int_{\Omega} u^{2}+\varepsilon \int_{\Omega}|\nabla u|^{2}\right)
$$

Taking account these estimations in (2.8) we get

$$
\frac{d}{d t} \int_{\Omega} u^{2}+(2-C \varepsilon) \int_{\Omega} u^{2} \leq(C \varepsilon-2) \int_{\Omega}|\nabla u|^{2}+C \int_{\Omega} 2|\nabla u||\nabla v|+C(\varepsilon) .
$$

Then,

$$
\frac{d}{d t} \int_{\Omega} u^{2}+(2-C \varepsilon) \int_{\Omega} u^{2} \leq((C+1) \epsilon-2) \int_{\Omega}|\nabla u|^{2}+C(\epsilon) \int_{\Omega}|\nabla v|^{2}+C(\varepsilon) .
$$

Multiplying this inequality by $e^{(2-C \varepsilon) t}$ we have

$$
\frac{d}{d t}\left(e^{(2-C \varepsilon) t} \int_{\Omega} u^{2}\right) \leq C(\varepsilon) e^{(2-C \varepsilon) t} \int_{\Omega}|\nabla v|^{2}+C e^{(2-C \varepsilon) t}
$$

and integrating in $(0, t)$ we obtain

$$
e^{(2-C \varepsilon) t} \int_{\Omega} u^{2}-\int_{\Omega} u_{0}^{2} \leq C(\varepsilon) \int_{0}^{t}\left(e^{(2-C \varepsilon) s} \int_{\Omega}|\nabla v|^{2}\right) d s+C \int_{0}^{t} e^{(2-C \varepsilon) s} d s .
$$

At this point, thanks to (2.7),

$$
\int_{\Omega} u^{2} \leq\left\|u_{0}\right\|^{2} e^{-(2-C \varepsilon) t}+C\left(\left\|v_{0}\right\|\right)+C
$$

and the Lemma is easily concluded. 
Lemma 2.4. Let $\gamma \in(1, \infty)$, and $t>t_{0}>0$ for $t_{0}$ small enough. If $\|u(t)\|_{L^{\gamma}(\Omega)} \leq C$, then

$$
\|v(t)\|_{W^{1, p(\gamma, n)}} \leq C
$$

where

$$
p(\gamma, n)=\gamma \frac{n}{n-1+\varepsilon \gamma} \quad \forall \varepsilon>0 .
$$

Before proving Lemma 2.4, we remind some facts about Sobolev spaces and the interpolation theory. For Sobolev spaces with non-integer index, it holds that ([3], pag 25)

$$
\text { If } s_{0}, s_{1} \in \mathbb{R}^{+} \backslash \mathbb{N}, s_{1} \leq s_{0} \Longrightarrow W^{s_{0}, p} \hookrightarrow W^{s_{1}, p} \forall p \in[1, \infty] .
$$

If the $p$-index is different, we can use the following general enough imbedding theorem

Theorem 2.5. ([1]. Theor 7.58) Let $\Omega \subset \mathbb{R}^{N}$ a $C^{2}$-domain. Let $s_{0}>0,1<p<q<\infty$ and $s_{1}=s_{0}-\frac{N}{p}+\frac{N}{q}$. If $s_{1} \geq 0$, then

$$
W_{p}^{s_{0}}(\Omega) \hookrightarrow W_{q}^{s_{1}}(\Omega) .
$$

With respect to the interpolation theory, we remind that if $E_{0}$ and $E_{1}$ are two normed spaces continuously embedded into a topological space $\mathcal{E}$, we can defined the real interpolation for $0<\theta<1$ and $1 \leq p \leq \infty$, which we denote $\left(E_{0}, E_{1}\right)_{\theta, p}$ (See Definition 22.1 of $[18])$. It is true that

1. $\left(E_{0}, E_{1}\right)_{\theta, p} \subset E_{0}+E_{1}$.

2. $E_{0} \cap E_{1} \hookrightarrow\left(E_{0}, E_{1}\right)_{\theta, p}$.

3. (Lemma 22.2) If $0<\theta<1$ and $1 \leq p \leq q \leq \infty$, then

$$
\left(E_{0}, E_{1}\right)_{\theta, p} \hookrightarrow\left(E_{0}, E_{1}\right)_{\theta, q} .
$$

Finally, because $\left(E_{0}, E_{1}\right)_{\theta, 1} \subset\left(E_{0}, E_{1}\right)_{\theta, p} \subset E_{0}+E_{1}$, it follows from Lemma 25.2 ii) of [18] that if $\left(E_{0}, E_{1}\right)_{\theta, p}$ is a Banach space, then

$$
\exists C>0 \text {, such that }\|a\|_{\left(E_{0}, E_{1}\right)_{\theta, p}} \leq C\|a\|_{E_{0}}^{1-\theta}\|a\|_{E_{1}}^{\theta} \quad \forall a \in E_{0} \cap E_{1} .
$$

The application of this theory for the Sobolev spaces is based on the following fundamental results. If we denote

$$
W_{\mathcal{B}}^{s, \gamma}=\left\{\begin{array}{ll}
W^{s, \gamma} & -1+1 / \gamma<s<1+1 / \gamma \\
\left(W^{-s, \gamma^{\prime}}\right)^{\prime} & -2+1 / \gamma<s \leq-1+1 / \gamma
\end{array},\right.
$$

then it holds

Theorem 2.6. ([3], Theor. 5.2; Theor. 7.2) Suppose that $(\mathcal{A}, \mathcal{B})$ is a normally elliptic Neumann problem on $\Omega$ with $C^{1}$-coefficients, $1<p<\infty$ and $0<\theta<1$. Then

1.

$$
\left(L_{p}, W_{\mathcal{B}}^{2, p}\right)_{\theta, p}=W_{\mathcal{B}}^{2 \theta, p}
$$

for $2 \theta \in(0,2) \backslash\{1,1+1 / p\}$. 
2. If $-2+1 / p<s_{0}<s_{1}<1+1 / p$, and $s_{\theta}:=(1-\theta) s_{0}+\theta s_{1} \notin \mathbb{N}$, then,

$$
\left(W_{\mathcal{B}}^{s_{0}, p}, W_{\mathcal{B}}^{s_{1}, p}\right)_{\theta, p}=W_{\mathcal{B}}^{s_{\theta}, p}
$$

When $(\mathcal{A}, \mathcal{B})$ is a normally elliptic problem on $\Omega$ with $C^{1}$-coefficients, it is possible to construct an interpolation-extrapolation scale of spaces; we put $E_{0}=L_{p}, E_{1}=W_{\mathcal{B}}^{2, p}$, and $E_{-1}$ a completion of $E_{0}$ (see [3], pag 29), we define

$$
E_{\theta}=\left(E_{0}, E_{1}\right)_{\theta, p}=W_{p, \mathcal{B}}^{2 \theta} \quad \text { for } \quad 2 \theta \in(0,2) \backslash\{1,1+1 / p\},
$$

and we can extend the definition inductively for $E_{k+\theta}$. Then, there exists a family of operators, $A_{\theta} \in \mathcal{L}\left(E_{1+\theta}, E_{\theta}\right)$, being $A_{\theta}$ the negative infinitesimal generator of an analytic semigroup on $E_{\theta}$. The semigroup $e^{-t A_{\theta}}$ is defined $e^{-t A_{\theta}}: E_{\theta} \rightarrow E_{\theta}$ (see [3], pags 28-30).

Proof of Lemma 2.4. System (2.3) has a local classical solution $(u(t), v(t))$ defined in $\left(0, T_{\max }\right)$. So, we pose the nonhomogeneous linear problem

$$
\left\{\begin{array}{cl}
\partial_{t} v-\Delta v+v=f(t):=-c u(t) v(t) & \text { in } \Omega \times(0, \infty) \\
\frac{\partial v}{\partial n}=0 & \text { on } \Gamma_{1} \times(0, T), \\
\frac{\partial v}{\partial n}=g(t):=\mu \frac{v(t)}{1+v(t)} & \text { on } \Gamma_{2} \times(0, T), \\
v(x, 0)=v_{0}(x), & \text { in } \Omega,
\end{array}\right.
$$

which we simply denote

$$
\left\{\begin{array}{cl}
\partial_{t} v-\mathcal{A}_{0} v=f(t) & \text { in } \Omega \times(0, \infty) \\
\mathcal{B}_{0} v=g(t) & \text { on } \Gamma \times(0, T), \\
v(x, 0)=v_{0}(x), & \text { in } \Omega,
\end{array}\right.
$$

$\left(\mathcal{A}_{0}, \mathcal{B}_{0}\right)$ generate an analytical semigroup whose generator is $A_{0} \in \mathcal{L}\left(L^{\gamma}(\Omega)\right)$ with $\operatorname{dom} A_{0}=$ $\operatorname{ker} \mathcal{B}$. The operator $\left.\mathcal{B}_{0}\right\rfloor_{\operatorname{Ker} \mathcal{A}_{0}}: \operatorname{Ker} \mathcal{A}_{0} \rightarrow W^{1-1 / \gamma, \gamma}(\partial \Omega)$ is an homeomorphism and we denote $\mathcal{B}_{0}^{c}$ its inverse operator. The generalized variation-of-constants formula gives, for $2 \alpha \in(1 / \gamma, 1+1 / \gamma)$

$$
v(t)=e^{-t A_{\alpha-1}} v_{0}-\int_{0}^{t} e^{-(t-\tau) A_{\alpha-1}}\left(f(\tau)+A_{\alpha-1} \mathcal{B}_{0}^{c} g(\tau)\right) d \tau,
$$

and (2.14) is well defined for $(f, g) \in C\left(\left(0, T_{\max }\right), W_{\gamma, \mathcal{B}_{0}}^{2 \alpha-2} \times \partial W_{\gamma}^{2 \alpha}\right)([3]$, pag 63). Note that it follows from (7.8) of [3] and (2.10) that for $-2 \leq s<0$,

$$
L^{\gamma} \hookrightarrow W_{\mathcal{B}_{0}}^{s, \gamma}
$$

We choose $\beta:=1+\frac{1}{\gamma}-\varepsilon<2 \alpha<1+\frac{1}{\gamma}$. Owing to Theorem 2.6, there exists some $0<\theta<1$ such that

So, it holds that

$$
\left(W_{\mathcal{B}_{0}}^{2 \alpha-2, \gamma}, W_{\mathcal{B}_{0}}^{2 \alpha, \gamma}\right)_{\theta, \gamma}=W_{\mathcal{B}_{0}}^{\beta, \gamma}=W^{\beta, \gamma} .
$$

$$
\forall w \in W_{\mathcal{B}_{0}}^{2 \alpha-2, \gamma} \cap W_{\mathcal{B}_{0}}^{2 \alpha, \gamma}=W_{\mathcal{B}_{0}}^{2 \alpha, \gamma}, \quad\|w\|_{W^{\beta, \gamma}} \leq C\|w\|_{W_{\mathcal{B}_{0}}^{2 \alpha, \gamma}}^{\theta}\|w\|_{W_{\mathcal{B}_{0}}^{2 \alpha-2, \gamma}}^{1-\theta} .
$$


If we remind (2.11), $W_{\mathcal{B}_{0}}^{2 \alpha, \gamma}=E_{\alpha}, W_{\mathcal{B}_{0}}^{2 \alpha-2, \gamma}=E_{\alpha-1}, A_{\alpha-1}: E_{\alpha} \rightarrow E_{\alpha-1}$ and the semigroup $e^{-t A_{\alpha-1}}: E_{\alpha-1} \rightarrow E_{\alpha-1}$. So, for $f \in W_{\mathcal{B}_{0}}^{2 \alpha-2, \gamma}=E_{\alpha-1}$,

$$
\left\|e^{-(t-\tau) A_{\alpha-1}} f\right\|_{W^{\beta, \gamma}} \leq C\left\|e^{-(t-\tau) A_{\alpha-1}} f\right\|_{W_{\mathcal{B}_{0}}^{2 \alpha, \gamma}}^{\theta}\left\|e^{-(t-\tau) A_{\alpha-1}} f\right\|_{W_{\mathcal{B}_{0}}^{2 \alpha-2}}^{1-\theta, \gamma}
$$

Because $A_{\alpha-1}$ is the generator of an analytic semigroup, it holds that ([3], Remark 8.6.c)

$$
\|w\|_{W_{\mathcal{B}_{0}}^{2 \alpha, \gamma}} \leq C\left\|A_{\alpha-1} w\right\|_{W_{\mathcal{B}_{0}}^{2 \alpha-2, \gamma}} \quad \forall w \in E_{\alpha}
$$

because $[0,+\infty) \subset \rho\left(-A_{0}\right)$; this claim holds following Theorem 8.5 and (3.1) of [3]. So

$$
\left\|e^{-(t-\tau) A_{\alpha-1}} f\right\|_{W^{\beta, \gamma}} \leq C\left\|A_{\alpha-1} e^{-(t-\tau) A_{\alpha-1}} f\right\|_{W_{\mathcal{B}_{0}}^{2 \alpha-2, \gamma}}^{\theta}\left\|e^{-(t-\tau) A_{\alpha-1}} f\right\|_{W_{\mathcal{B}_{0}}^{2 \alpha-2, \gamma}}^{1-\theta} .
$$

Finally, thanks to [10], Theor 1.3.4,

$$
\begin{gathered}
\left\|A_{\alpha-1} e^{-(t-\tau) A_{\alpha-1}} f\right\|_{W_{\mathcal{B}_{0}}^{2 \alpha-2, \gamma}}^{\theta} \leq C(t-\tau)^{-\theta} e^{-\delta(t-\tau) \theta}\|f\|_{W_{\mathcal{B}_{0}}^{2 \alpha-2, \gamma}}^{\theta}, \\
\left\|e^{-(t-\tau) A_{\alpha-1}} f\right\|_{W_{\mathcal{B}_{0}}^{2-\theta}}^{1-\theta} \leq C e^{-\delta(t-\tau)(1-\theta)}\|f\|_{W_{\mathcal{B}_{0}}^{2 \alpha-2, \gamma}}^{1-\theta},
\end{gathered}
$$

and it results

$$
\left\|e^{-(t-\tau) A_{\alpha-1}} f\right\|_{W^{\beta, \gamma}} \leq C e^{-\delta(t-\tau)}(t-\tau)^{-\theta}\|f\|_{W_{\mathcal{B}_{0}}^{2 \alpha-2, \gamma}}
$$

for each $\delta \in(0,1)$. Taking norm $\|\cdot\|_{W^{\beta, \gamma}}$ on both sides of (2.14) and using (2.16), we get

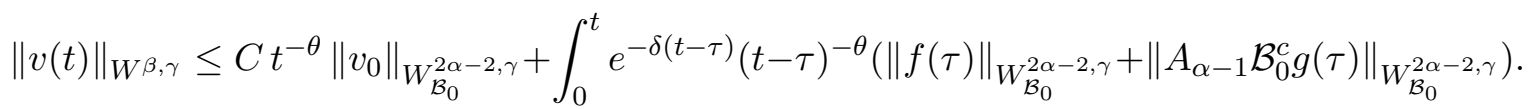

Taking into account Lemma 2.2, (2.15) and the Sobolev embedding $W^{\beta, \gamma} \hookrightarrow W^{1, p(n, \gamma)}$, we deduce easily the Lemma.

Lemma 2.7. Given any $\alpha \in[1,+\infty)$, if $\left\|u_{0}\right\|_{L^{\alpha}(\Omega)}<+\infty$ then there exists $C>0$ (depending on $\alpha$ and $\left\|u_{0}\right\|_{L^{\alpha}(\Omega)}$ ) such that $u(t) \in L^{\alpha}(\Omega)$ and

$$
\|u(t)\|_{L^{\alpha}(\Omega)} \leq C \quad \forall t>t_{0} .
$$

Proof. Fix $\alpha>2$. Multiplying the $u$-equation by $\alpha u^{\alpha-1}$ and integrating in $\Omega$, we obtain

$$
\begin{aligned}
\frac{d}{d t} \int_{\Omega} u^{\alpha}= & \frac{-4(\alpha-1)}{\alpha} \int_{\Omega}\left|\nabla\left(u^{\alpha / 2}\right)\right|^{2}+\alpha(\alpha-1) \int_{\Omega} V(u) u^{\alpha-2} \nabla v \cdot \nabla u- \\
& -\alpha \int_{\partial \Omega} V(u) \partial_{n} v u^{\alpha-1}+\lambda \alpha \int_{\Omega} u^{\alpha}-\alpha \int_{\Omega} u^{\alpha+1} .
\end{aligned}
$$

We add $\int_{\Omega} u^{\alpha}$ on both sides of the equality. Besides, we estimate the term $-\alpha \int_{\partial \Omega} V(u) \partial_{n} v u^{\alpha-1}$.

$$
-\alpha \int_{\Gamma_{2}} V(u) \mu \frac{v}{1+v} u^{\alpha-1} \leq C \int_{\Gamma_{2}} u^{\alpha-1}
$$

At this point, we use the following inequality. Given $\varepsilon>0$, there exists $C(\varepsilon)>0$ such that

$$
s^{\alpha-1} \leq \varepsilon s^{\alpha}+C(\varepsilon) \quad \forall s \in \mathbf{R} .
$$


Then,

$$
\begin{gathered}
\int_{\Gamma_{2}} u^{\alpha-1} \leq \varepsilon \int_{\Gamma_{2}} u^{\alpha}+C(\varepsilon)\left|\Gamma_{2}\right|= \\
=\varepsilon \int_{\Gamma_{2}}\left(u^{\alpha / 2}\right)^{2}+C \leq C\left(\varepsilon \int_{\Omega}\left(u^{\alpha / 2}\right)^{2}+\varepsilon \int_{\Omega}\left|\nabla\left(u^{\alpha / 2}\right)\right|^{2}\right)+C,
\end{gathered}
$$

using the injection $H^{1}(\Omega)$ in $L^{2}\left(\Gamma_{2}\right)$. Taking account this estimation in $(2.17)$, we have

$$
\begin{gathered}
\frac{d}{d t} \int_{\Omega} u^{\alpha}+\int_{\Omega} u^{\alpha} \leq \\
\leq \frac{-4(\alpha-1)}{\alpha} \int_{\Omega}\left|\nabla\left(u^{\alpha / 2}\right)\right|^{2}+\alpha(\alpha-1) \int_{\Omega} V(u) u^{\alpha-2} \nabla v \cdot \nabla u+\int_{\Omega}\left((\alpha \lambda+1) u^{\alpha}-\alpha u^{\alpha+1}\right)+ \\
+C \varepsilon \int_{\Omega} u^{\alpha}+C \varepsilon \int_{\Omega}\left|\nabla\left(u^{\alpha / 2}\right)\right|^{2}+C .
\end{gathered}
$$

Using $(\alpha \lambda+1) s^{\alpha}-\alpha s^{\alpha+1} \leq C=C(\lambda, \alpha)$ for every $s \geq 0$,

$$
\begin{gathered}
\frac{d}{d t} \int_{\Omega} u^{\alpha}+(1-C \varepsilon) \int_{\Omega} u^{\alpha} \leq \\
\leq\left(\frac{-4(\alpha-1)}{\alpha}+C \varepsilon\right) \int_{\Omega}\left|\nabla\left(u^{\alpha / 2}\right)\right|^{2}+\alpha(\alpha-1) \int_{\Omega} V(u) u^{\alpha-2} \nabla v \cdot \nabla u+C .
\end{gathered}
$$

An easy computation gives

$$
\alpha(\alpha-1) \int_{\Omega} V(u) u^{\alpha-2} \nabla v \cdot \nabla u=2(\alpha-1) \int_{\Omega} u^{\frac{\alpha}{2}-1} V(u) \nabla v \cdot \nabla\left(u^{\alpha / 2}\right)
$$

and replacing it in the previous inequality, we obtain

$$
\begin{gathered}
\frac{d}{d t} \int_{\Omega} u^{\alpha}+(1-\varepsilon) \int_{\Omega} u^{\alpha} \leq \\
\leq\left(\frac{-4(\alpha-1)}{\alpha}+\varepsilon\right) \int_{\Omega}\left|\nabla\left(u^{\alpha / 2}\right)\right|^{2}+2(\alpha-1) \int_{\Omega} u^{\frac{\alpha}{2}-1} V(u) \nabla v \cdot \nabla\left(u^{\alpha / 2}\right)+C .
\end{gathered}
$$

Now, we deal with the second term in the right hand side,

$$
\begin{gathered}
\left\|u^{\frac{\alpha}{2}-1} V(u) \nabla v \cdot \nabla\left(u^{\alpha / 2}\right)\right\|_{1} \leq C\left\|u^{\frac{\alpha}{2}-1}\right\|_{L^{\theta(p)}(\Omega)}\|\nabla v\|_{L^{p}(\Omega)}\left\|\nabla\left(u^{\alpha / 2}\right)\right\|_{L^{2}(\Omega)} \leq \\
\leq C(\varepsilon)\left\|u^{\frac{\alpha}{2}-1}\right\|_{L^{\theta(p)}(\Omega)}^{2}\|\nabla v\|_{L^{p}(\Omega)}^{2}+\varepsilon\left\|\nabla\left(u^{\alpha / 2}\right)\right\|_{L^{2}(\Omega)}^{2},
\end{gathered}
$$

where

$$
\theta(p)=\frac{2 p}{p-2}, p>2 .
$$

Replacing (2.19) into (2.18), and considering $\varepsilon$ small enough, we obtain

$$
\frac{d}{d t}\left\|u^{\alpha / 2}\right\|_{L^{2}(\Omega)}^{2}+(1-C \varepsilon)\left\|u^{\alpha / 2}\right\|_{L^{2}(\Omega)}^{2} \leq C(\varepsilon)\left\|u^{\frac{\alpha}{2}-1}\right\|_{L^{\theta(p)}(\Omega)}^{2}\|\nabla v\|_{L^{p}(\Omega)}^{2}+C .
$$

Now, we begin a recursive algorithm. Taking

$$
\gamma_{0}:=2
$$


by Lemma $2.3 u(t) \in L^{2}(\Omega),\|u(t)\|_{L^{2}(\Omega)} \leq C$. By Lemma 2.4, $v(t) \in W^{1, p\left(\gamma_{0}, N\right)}(\Omega)$, so, $\|\nabla v\|_{L^{p}(\Omega)}^{2}$ is finite. Choosing $\alpha \leq 2+\frac{4}{\theta(p)}$ we assure that $u^{\frac{\alpha}{2}-1} \in L^{\theta}(\Omega)$. So, for $2<\alpha \leq 2+\frac{2 \gamma_{0}}{\theta\left(p\left(\gamma_{0}, N\right)\right)}$ we have

$$
\frac{d}{d t}\left\|u^{\alpha / 2}(t)\right\|_{L^{2}(\Omega)}^{2}+(1-\varepsilon)\left\|u^{\alpha / 2}(t)\right\|_{L^{2}(\Omega)}^{2} \leq C .
$$

Therefore,

$$
\left\|u^{\alpha / 2}(t)\right\|_{L^{2}(\Omega)}^{2} \leq\left\|u_{0}^{\alpha / 2}\right\|_{L^{2}(\Omega)}^{2}+C .
$$

We have just proved that $\|u(t)\|_{L^{\alpha}(\Omega)} \leq C$ for $2<\alpha \leq 2+\frac{2 \gamma_{0}}{\theta\left(p\left(\gamma_{0}, N\right)\right)}$.

Now, we define

$$
\gamma_{1}:=2+\frac{2 \gamma_{0}}{\theta\left(p\left(\gamma_{0}, N\right)\right)}
$$

Owing to the previous reasoning, we have that $u(t) \in L^{\gamma_{1}}(\Omega)$. So, by Lemma $2.4 v(t) \in$ $W^{1, p\left(\gamma_{1}, N\right)}(\Omega)$. If we choose $\alpha \leq 2+\frac{2 \gamma_{1}}{\theta\left(p\left(\gamma_{1}, N\right)\right)}$ we will assure that $u^{\alpha / 2-1}(t) \in L^{\theta}(\Omega)$, and by $(2.20)$ we obtain that for $2<\alpha \leq 2+\frac{2 \gamma_{1}}{\theta\left(p\left(\gamma_{1}, N\right)\right)}, u(t) \in L^{\alpha}(\Omega)$ and $\|u(t)\|_{L^{\alpha}(\Omega)} \leq C$.

By a recursive algorithm we get that $u(t) \in L^{\alpha}(\Omega)$ and $\|u(t)\|_{L^{\alpha}(\Omega)} \leq C$, for every $\alpha$ with $2<\alpha \leq 2+\frac{2 \gamma_{n}}{\theta\left(p\left(\gamma_{n}, N\right)\right)}$, and

$$
\gamma_{n}=2+\frac{2 \gamma_{n-1}}{\theta\left(p\left(\gamma_{n-1}, N\right)\right)}
$$

Using that

$$
p\left(\gamma_{n-1}, N\right)=\gamma_{n-1} \frac{N}{N-1+\varepsilon \gamma_{n-1}},
$$

we have that

$$
\gamma_{n}=\gamma_{n-1}\left(1-\frac{2 \varepsilon}{N}\right)+\frac{2}{N} .
$$

The limit of $\gamma_{n}$ is $\frac{1}{\varepsilon}$, so for $\varepsilon>0$ as small as we want, we have that $u(t) \in L^{\alpha}(\Omega)$ and $\|u(t)\|_{L^{\alpha}(\Omega)} \leq C$ for all $\alpha \in[1,+\infty)$.

Remark 2.8. Consequently, by Lemmas 2.4 and 2.7, we have obtained that $u(t) \in$ $L^{p}(\Omega), \nabla v \in L^{p}(\Omega)^{N}$, for every $p \geq 1$.

In the following result we obtain a better bound of $u$, a $L^{\infty}$-bound. Let $p>1$ and define

$$
B:=-\Delta+I,
$$

with domain

$$
D(B):=\left\{u \in W^{2, p}(\Omega): \frac{\partial u}{\partial n}=0 \text { on } \partial \Omega\right\}
$$

For each $\beta \geq 0$ define

$$
X^{\beta}:=D\left(B^{\beta}\right) \quad \text { with the norm }\|u\|_{\beta}:=\left\|B^{\beta} u\right\|_{p} .
$$

Lemma 2.9. Let $2 \beta<1$, then for $t>t_{0}>0$ we have

$$
\|u(t)\|_{\beta} \leq C
$$


Proof. We have that

$$
u(t)=e^{-t B} u_{0}+\int_{0}^{t} e^{-(t-\tau) B}\left(-\nabla \cdot(V(u) \nabla v)+(\lambda+1) u-u^{2}\right) d \tau,
$$

and so

$$
\|u(t)\|_{\beta} \leq\left\|e^{-t B} u_{0}\right\|_{\beta}+\int_{0}^{t}\left\|e^{-(t-\tau) B}\left(-\nabla \cdot(V(u) \nabla v)+(\lambda+1) u-u^{2}\right) d \tau\right\|_{\beta} .
$$

First, by [10, Theorem 1.4.3]

$$
\left\|e^{-t B} u_{0}\right\|_{\beta} \leq C t^{-\beta} e^{-\delta t}\left\|u_{0}\right\|_{p}
$$

and

$$
\left\|e^{-(t-\tau) B}\left(\lambda u-u^{2}\right)\right\|_{\beta} \leq(t-\tau)^{-\beta} e^{-\delta(t-\tau)}\left((\lambda+1)\|u\|_{p}+\left\|u^{2}\right\|_{p}\right)
$$

where $\delta \in(0,1)$.

Moreover, by [13, Lemma 2.1] we obtain

$$
\begin{aligned}
\| e^{-(t-\tau) B}\left(-\nabla \cdot(V(u) \nabla v) \|_{\beta}\right. & \leq C \| e^{-(t-\tau) \Delta}\left(-\nabla \cdot(V(u) \nabla v) \|_{\beta}\right. \\
& \leq C(\varepsilon)(t-\tau)^{-1 / 2-\beta-\epsilon} e^{-\delta(t-\tau)}\|V(u) \nabla v\|_{p}
\end{aligned}
$$

where $\epsilon>0$ such that $-1 / 2-\beta-\epsilon>-1$. Then,

$$
\begin{aligned}
\|u(t)\|_{\beta} \leq & C t^{-\beta} e^{-\delta t}\left\|u_{0}\right\|_{p}+C \int_{0}^{t}\left[(t-\tau)^{-1 / 2-\beta-\epsilon} e^{-\delta(t-\tau)}\|V(u) \nabla v\|_{p}+\right. \\
& \left.(t-\tau)^{-\beta} e^{-\delta(t-\tau)}\left((\lambda+1)\|u\|_{p}+\left\|u^{2}\right\|_{p}\right)\right] d \tau
\end{aligned}
$$

Now, observe that

$$
\|V(u) \nabla v\|_{p} \leq C\|\nabla v\|_{L^{p}(\Omega)} .
$$

Finally, thanks to Lemmas 2.4 and 2.7 we easily conclude the result from (2.21).

Corollary 2.10. We have that

$$
\|u(t)\|_{\infty} \leq C \text { for all } t \geq 0
$$

and consequently we have proved the global existence.

Proof. Let $p>N, 2 \beta \in\left(\frac{N}{p}, 1\right)$. Since $2 \beta>N / p$ we have by [10, Theorem 1.6.1] that

$$
X^{\beta} \hookrightarrow C(\bar{\Omega})
$$

Thanks to Lemma 2.9 we have that $\|u(t)\|_{\infty}<C$ for $t>t_{0}>0$. Moreover, the local existence Theorem yields $\|u(t)\|_{\infty}<C$ for $t<t_{0}$. Therefore, $\|u(t)\|_{\infty}<C$ for all $t \geq 0$. Then, this result and Lemma 2.2 prove the global existence criterium (see (2.2)). 


\section{Steady-states}

Consider now the stationary problem

$$
\begin{cases}-\Delta u=-\operatorname{div}(V(u) \nabla v)+\lambda u-u^{2} & \text { in } \Omega, \\ -\Delta v=-v-c u v & \text { in } \Omega, \\ \frac{\partial u}{\partial n}=\frac{\partial v}{\partial n}=0 & \text { on } \Gamma_{1}, \\ \frac{\partial u}{\partial n}=0, \quad \frac{\partial v}{\partial n}=\mu \frac{v}{1+v} & \text { on } \Gamma_{2} .\end{cases}
$$

First, we need to introduce some notations.

For $\alpha \in(0,1)$ we denote

$$
X_{1}:=\left\{u \in C^{2, \alpha}(\bar{\Omega}): \partial u / \partial n=0 \text { on } \partial \Omega\right\}, \quad X_{2}:=\left\{u \in C^{2, \alpha}(\bar{\Omega}): \partial u / \partial n=0 \text { on } \Gamma_{1}\right\}
$$

and finally

$$
X:=X_{1} \times X_{2} .
$$

Moreover, given a function $c \in C(\bar{\Omega})$ we denote by

$$
c_{M}:=\max _{\bar{\Omega}} c(x), \quad c_{L}:=\min _{\bar{\Omega}} c(x) .
$$

We are interested in solutions $(u, v) \in X$ of (3.1) with both components non-negative and non-trivial. Observe that thanks to the strong maximum principle, any component, $u$ or $v$, of a non-negative and non-trivial solution is in fact positive in the whole domain $\bar{\Omega}$.

Consider functions $m \in C^{\alpha}(\bar{\Omega}), g \in C^{1, \alpha}\left(\Gamma_{2}\right)$ and the eigenvalue problem

$$
\begin{cases}-\Delta \phi+m \phi=\lambda \phi & \text { in } \Omega, \\ \frac{\partial \phi}{\partial n}=0 & \text { on } \Gamma_{1} \\ \frac{\partial \phi}{\partial n}+g \phi=0 & \text { on } \Gamma_{2} .\end{cases}
$$

We are interested only in the principal eigenvalue of (3.2), i.e., the eigenvalues which have an associated positive eigenfunction. In the following result we recall its main properties, see [4], [5] and [8].

Lemma 3.1. Problem (3.2) admits a unique principal eigenvalue, which will be denoted by $\lambda_{1}(-\Delta+m ; N, N+g)$. Moreover, this eigenvalue is simple, and any positive eigenfunction, $\phi$, verifies $\phi \in C_{0}^{1, \alpha}(\bar{\Omega})$. In addition, $\lambda_{1}(-\Delta+m ; N, N+g)$ is separately increasing in $m$ and $g$; and when $g=K$ constant, it verifies

$$
\begin{aligned}
& \lim _{K \rightarrow-\infty} \lambda_{1}(-\Delta+m ; N, N+K)=-\infty, \\
& \lim _{K \rightarrow+\infty} \lambda_{1}(-\Delta+m ; N, N+K)=\lambda_{1}(-\Delta+m ; N, D),
\end{aligned}
$$

where $\lambda_{1}(-\Delta+m ; N, D)$ stands for the principal eigenvalue of $-\Delta+m$ with homogeneous Dirichlet boundary conditions on $\Gamma_{2}$ and $\partial \phi / \partial n=0$ on $\Gamma_{1}$. 
Moreover, we are going to consider the following Steklov eigenvalue problem with eigenvalue on the boundary

$$
\begin{cases}-\Delta \phi+m \phi=0 & \text { in } \Omega, \\ \frac{\partial \phi}{\partial n}=0 & \text { on } \Gamma_{1} \\ \frac{\partial \phi}{\partial n}+g \phi=\mu \phi & \text { on } \Gamma_{2}\end{cases}
$$

It is well-known that there exists a principal eigenvalue of (3.4), we denote it by

$$
\mu_{1}(-\Delta+m ; N, N+g) .
$$

It is clear that $\mu$ is a principal eigenvalue of (3.4) if, and only if,

$$
0=\lambda_{1}(-\Delta+m ; N, N+g-\mu),
$$

and that

$$
0>\lambda_{1}(-\Delta+m ; N, N+g-\mu) \Longleftrightarrow \mu>\mu_{1}(-\Delta+m ; N, N+g),
$$

and analogously,

$$
0<\lambda_{1}(-\Delta+m ; N, N+g-\mu) \Longleftrightarrow \mu<\mu_{1}(-\Delta+m ; N, N+g) .
$$

\subsection{Semi-trivial solutions}

Apart from the trivial solution $(u, v)=(0,0)$ of $(3.1)$, there exist the semi-trivial solutions. It is clear that if $v \equiv 0$, then $u$ verifies

$$
-\Delta u=\lambda u-u^{2} \quad \text { in } \Omega, \quad \partial u / \partial n=0 \quad \text { on } \partial \Omega,
$$

that is $u \equiv \lambda$.

On the other hand, when $u \equiv 0$ then $v$ satisfies the equation

$$
\begin{cases}-\Delta v+v=0 & \text { in } \Omega, \\ \frac{\partial v}{\partial n}=0 & \text { on } \Gamma_{1}, \\ \frac{\partial v}{\partial n}=\mu \frac{v}{1+v} & \text { on } \Gamma_{2} .\end{cases}
$$

This equation was analyzed in [19] with $\Gamma_{1}=\emptyset$, we include a proof for reader's convenience and some useful estimates.

Proposition 3.2. There exists a positive solution of (3.5) if, and only if,

$$
\mu>\mu_{1}:=\mu_{1}(-\Delta+1 ; N, N) .
$$

Moreover, if the solution exists, it is the unique positive solution, and we denote it by $\theta_{\mu}$. Furthermore, $\theta_{\mu}$ is locally asymptotically stable (l. a. s.) for $\mu>\mu_{1}$, i.e.,

$$
\lambda_{1}\left(-\Delta+1 ; N, N-\mu\left(1 /\left(1+\theta_{\mu}\right)\right)^{2}\right)>0 .
$$

Finally,

$$
\frac{1}{\left\|\varphi_{1}\right\|_{\infty}}\left(\frac{\mu}{\mu_{1}}-1\right) \varphi_{1} \leq \theta_{\mu} \leq \frac{1}{\left(\varphi_{1}\right)_{L}}\left(\frac{\mu}{\mu_{1}}-1\right) \varphi_{1}, \quad \text { in } \Omega,
$$

where $\varphi_{1}$ is a positive eigenfunction associated to $\mu_{1}$. 
Proof. Observe that if $v$ is a positive solution of (3.5) we get

$$
0=\lambda_{1}\left(-\Delta+1 ; N, N-\mu \frac{1}{1+v}\right),
$$

and so $\mu>0$. Moreover,

$$
0=\lambda_{1}\left(-\Delta+1 ; N, N-\mu \frac{1}{1+v}\right)>\lambda_{1}(-\Delta+1 ; N, N-\mu)
$$

and then, $\mu>\mu_{1}$.

To prove the existence of solution, we apply the sub-supersolution method. Take $\varphi_{1}$ a positive eigenfunction associated to $\mu_{1}$. Then $(\underline{v}, \bar{v})=\left(\varepsilon \varphi_{1}, M \varphi_{1}\right)$ is sub-supersolution of (3.5) if

$$
\varepsilon=\frac{\frac{\mu}{\mu_{1}}-1}{\left\|\varphi_{1}\right\|_{\infty}} \quad \text { and } \quad K \geq \frac{\frac{\mu}{\mu_{1}}-1}{\left(\varphi_{1}\right)_{L}} .
$$

The uniqueness follows by an standard argument. Indeed, observe that the map $s \mapsto$ $\mu s / s(1+s)=\mu /(1+s)$ is decreasing.

To prove the stability, linearizing (3.5) around $\theta_{\mu}$, we need to prove that

$$
\lambda_{1}\left(-\Delta+1 ; N, N-\mu\left(1 /\left(1+\theta_{\mu}\right)\right)^{2}\right)>0 .
$$

For that, observe that $\bar{v}=\theta_{\mu}$ is a strict-supersolution of

$$
-\Delta v+v=0 \quad \text { in } \Omega, \quad \frac{\partial v}{\partial n}=0 \quad \text { on } \Gamma_{1}, \quad \frac{\partial v}{\partial n}-\mu\left(\frac{1}{1+\theta_{\mu}}\right)^{2} v=0 \quad \text { on } \Gamma_{2} .
$$

The next result provides us with a priori bounds of the solutions of (3.1) and bounds in the space $X$.

Lemma 3.3. Let $(u, v)$ a coexistence state of (3.1). Then,

$$
u \leq \lambda \text { and } v \leq \theta_{\mu} .
$$

Moreover, consider that $(\lambda, \mu) \in \mathcal{K} \subset \mathbb{R}^{2}$ compact. Then, there exists a constant $C$ (independent of $\lambda$ and $\mu$ ) such that for any solution $(u, v)$ of (3.1) we have

$$
\|(u, v)\|_{X} \leq C .
$$

Proof. That $v \leq \theta_{\mu}$ is clear. On the other hand, observe that the first equation of (3.1) can be written as

$$
-\Delta u=-V^{\prime}(u) \nabla u \cdot \nabla v-V(u) \Delta v+\lambda u-u^{2}=-V^{\prime}(u) \nabla u \cdot \nabla v-V(u)(v+c u v)+\lambda u-u^{2} .
$$

Then, if we denote by $\bar{x} \in \Omega$ such that $u(\bar{x})=\max _{\bar{\Omega}} u$, using that $-\Delta u(\bar{x}) \geq 0$ and $\nabla u(\bar{x})=0$, we get

$$
u(\bar{x}) \leq \lambda-\frac{V(u(\bar{x}))}{u(\bar{x})} v(\bar{x})[1+c u(\bar{x})] .
$$

Then we can conclude that $u \leq \lambda$. This completes the proof of (3.8). 
Suppose $(\lambda, \mu) \in \mathcal{K} \subset \mathbb{R}^{2}$ compact and let $(u, v)$ be a solution of (3.1). Then, we have that $u$ and $v$ are bounded in $L^{\infty}(\Omega)$ for some constant $C$ not depending on $\lambda$ or $\mu$. Now, going back to the $v$-equation and using the $L^{p}$-estimates of Agmon, Douglis and Nirenberg [2], we have that for $p$ large

$$
\|v\|_{C^{1}(\bar{\Omega})} \leq C\|v\|_{W^{2, p}(\Omega)} \leq C\left(\|-v-c u v\|_{p}+\|\mu v /(1+v)\|_{p}\right) \leq C .
$$

But, the $u$-equation in (3.1) can be written as follows

$$
-\Delta u+V^{\prime}(u) \nabla u \cdot \nabla v=\lambda u-u^{2}-V(u)(v+c u v)
$$

and thus, $u$ is bounded in $W^{2, p}(\Omega)$ for all $p>1$, and so in $C^{1}(\bar{\Omega})$. Now, again using the $v$-equation and the Schauder Theory in Hölder spaces (see [9]), $v$ is bounded in $X_{2}$, and finally $u$ in $X_{1}$ with constants independent of $\lambda$ and $\mu$.

As an easy consequence of the above result, we have

Corollary 3.4. If $\lambda \leq 0$ or $\mu \leq \mu_{1}$ then (3.1) does not possess positive solution.

The following result will be crucial in the existence result:

Proposition 3.5. 1. Assume that $V^{\prime}(0)>0$ and fix $\lambda>0$. Then, there exists $\mu_{0}(\lambda)$ such that (3.1) does not possess coexistence states for $\mu \geq \mu_{0}(\lambda)$.

2. Fix $\mu>\mu_{1}$. Then, there exists $\lambda_{0}(\mu)$ such that (3.1) does not possess coexistence states for $\lambda \geq \lambda_{0}(\mu)$.

Proof. 1. Fix $\lambda>0$ and assume that there exist a sequence $\mu_{n} \rightarrow \infty$ and coexistence states $\left(u_{n}, v_{n}\right)$ of (3.1). Denote by $x_{n} \in \Omega$ such that $u_{n}\left(x_{n}\right)=\left\|u_{n}\right\|_{\infty}$. Then, by (3.9) we have

$$
\left\|u_{n}\right\|_{\infty}+\frac{V\left(\left\|u_{n}\right\|_{\infty}\right)}{\left\|u_{n}\right\|_{\infty}} v_{n}\left(x_{n}\right)\left(1+c\left\|u_{n}\right\|_{\infty}\right) \leq \lambda .
$$

Moreover, we know that $u_{n} \leq \lambda$, and so

$$
-\Delta v_{n}+(1+c \lambda) v_{n} \geq 0
$$

and so by a similar argumento to the proof of Proposition 3.2, we get that

$$
v_{n}(x) \geq\left(\frac{\mu_{n}}{\mu_{1}}-1-c \lambda\right) \varphi_{1}(x),
$$

and so by (3.10), we have

$$
\left\|u_{n}\right\|_{\infty}+\frac{V\left(\left\|u_{n}\right\|_{\infty}\right)}{\left\|u_{n}\right\|_{\infty}}\left(1+c\left\|u_{n}\right\|_{\infty}\right)\left(\frac{\mu_{n}}{\mu_{1}}-1-c \lambda\right) \varphi_{1}\left(x_{n}\right) \leq \lambda .
$$

Since $\varphi_{1} \geq \delta>0$ in $\bar{\Omega}$ and $1+c\left\|u_{n}\right\|_{\infty} \geq 1$, we obtain that $V\left(\left\|u_{n}\right\|_{\infty}\right) /\left\|u_{n}\right\|_{\infty} \rightarrow 0$, which is impossible due to $V^{\prime}(0)>0$.

2 . Denote by $u_{m}=\min _{x \in \Omega} u(x)$. Since the minimum can not attain at the boundary (because in such case $\partial u\left(x_{m}\right) / \partial n<0$ ) then, using again that $\nabla u\left(x_{m}\right)=0$ and $-\Delta u\left(x_{m}\right) \leq$ 0 we get

$$
\lambda \leq u_{m}+\frac{V\left(u_{m}\right)}{u_{m}} v\left(x_{m}\right)\left(1+c u_{m}\right) .
$$


Since $v \leq \theta_{\mu}$, if $\lambda \rightarrow \infty$ then $u_{m} \rightarrow \infty$. On the other hand, since $v$ is solution of the second equation of (3.1) we have

$$
0=\lambda_{1}\left(-\Delta+1+c u ; N, N-\frac{\mu}{1+v}\right) \geq \lambda_{1}\left(-\Delta+1+c u_{m} ; N, N-\mu\right) .
$$

But observe that $\left.\lambda_{1}\left(-\Delta+1+c u_{m}\right) ; N, N-\mu\right) \rightarrow \infty$ as $\lambda \rightarrow \infty$, a contradiction.

Finally, another eigenvalue problem is analyzed

$$
\begin{cases}-\Delta \phi+\operatorname{div}\left(V^{\prime}(0) \nabla \theta_{\mu} \phi\right)=\lambda \phi & \text { in } \Omega, \\ \frac{\partial \phi}{\partial n}=0 & \text { on } \Gamma_{1} \cup \Gamma_{2} .\end{cases}
$$

Fix $\mu>\mu_{1}$, we denote the principal eigenvalue as $\Lambda(\mu)$ and extend $\Lambda(\mu)=0$ for $\mu \leq \mu_{1}$. Observe that $\Lambda(\mu)=0$ when $V^{\prime}(0)=0$. In the following result we show some properties of $\Lambda(\mu)$.

Proposition 3.6. Assume that $V^{\prime}(0)>0$. Then

$$
\lim _{\mu \rightarrow \infty} \Lambda(\mu)=\infty
$$

Proof. Under a change of variable $\Phi=e^{\frac{V^{\prime}(0)}{2} \phi}$, we get

$$
\Lambda(\mu)=\lambda_{1}\left(-\Delta+\frac{V^{\prime}(0)^{2}}{4}\left|\nabla \theta_{\mu}\right|^{2}+\frac{V^{\prime}(0)}{2} \theta_{\mu} ; N, N+\frac{V^{\prime}(0)}{2} \mu \frac{\theta_{\mu}}{1+\theta_{\mu}}\right) .
$$

And so, using (3.7) we obtain that

$$
\Lambda(\mu)>\lambda_{1}\left(-\Delta+\frac{V^{\prime}(0)}{2} \frac{\frac{\mu}{\mu_{1}}-1}{\left\|\varphi_{1}\right\|_{\infty}} \varphi_{1} ; N, N\right) \rightarrow \infty
$$

as $\mu \rightarrow \infty$.

Now, finally we denote by

$$
\mu_{1}(\lambda)=\mu_{1}(-\Delta+1+c \lambda ; N, N)
$$

The main result is:

Theorem 3.7. 1. Assume that $V^{\prime}(0)>0$. Then there exists at least a coexistence state if

$$
(\lambda-\Lambda(\mu))\left(\mu-\mu_{1}(\lambda)\right)>0
$$

2. Assume that $V^{\prime}(0)=0$. Then there exists at least a coexistence state if $\lambda>0$ and

$$
\mu>\mu_{1}(\lambda)
$$


Proof. We fix $\mu>\mu_{1}$ and consider $\lambda$ as bifurcation parameter. First, we apply the Crandall-Rabinowitz theorem [6] in order to find the bifurcation point from the semitrivial solution $\left(0, \theta_{\mu}\right)$. Consider the map $\mathcal{F}: \mathbb{R} \times X_{1} \times X_{2} \mapsto C^{\alpha}(\bar{\Omega}) \times C^{\alpha}(\bar{\Omega}) \times C^{\alpha}\left(\Gamma_{2}\right)$ defined by

$$
\mathcal{F}(\lambda, u, v):=\left(-\Delta u+\operatorname{div}(V(u) \nabla v)-\lambda u+u^{2},-\Delta v+v+c u v, \frac{\partial v}{\partial n}-\mu \frac{v}{1+v}\right) .
$$

It is clear that $\mathcal{F}$ is regular, that $\mathcal{F}\left(\lambda, 0, \theta_{\mu}\right)=0$ and

$$
D_{(u, v)} \mathcal{F}\left(\lambda_{0}, u_{0}, v_{0}\right)\left(\begin{array}{c}
\xi \\
\eta
\end{array}\right)=\left(\begin{array}{c}
-\Delta \xi+\operatorname{div}\left(V^{\prime}\left(u_{0}\right) \xi \nabla v_{0}+V\left(u_{0}\right) \nabla \eta\right)-\lambda \xi+2 u_{0} \xi \\
-\Delta \eta+\eta+c u_{0} \eta+c v_{0} \xi \\
\frac{\partial \eta}{\partial n}-\mu\left(\frac{1}{1+v_{0}}\right)^{2} \eta
\end{array}\right)
$$

Hence, for $\left(u_{0}, v_{0}\right)=\left(0, \theta_{\mu}\right)$ and $\lambda_{0}=\Lambda(\mu)$ and we get that

$$
\operatorname{Ker}\left[D_{(u, v)} \mathcal{F}\left(\lambda_{0}, 0, \theta_{\mu}\right)\right]=\operatorname{span}\left\{\left(\Phi_{1}, \Phi_{2}\right)\right\}
$$

where $\Phi_{1}$ is an eigenfunction associated to $\Lambda(\mu)$ and $\Phi_{2}$ is the unique solution of

$$
\begin{cases}(-\Delta+1) \Phi_{2}=-c \theta_{\mu} \Phi_{1} & \text { in } \Omega \\ \frac{\partial \Phi_{2}}{\partial n}=0 & \text { on } \Gamma_{1} \\ \frac{\partial \Phi_{2}}{\partial n}-\mu\left(1 /\left(1+\theta_{\mu}\right)\right)^{2} \Phi_{2}=0 & \text { on } \Gamma_{2}\end{cases}
$$

Observe that $\Phi_{2}$ is well-defined by (3.6). Hence, $\operatorname{dim}\left(\operatorname{Ker}\left[D_{(u, v)} \mathcal{F}\left(\lambda_{0}, 0, \theta_{\mu}\right)\right]\right)=1$.

On the other hand, observe that

$$
D_{\lambda(u, v)} \mathcal{F}\left(\lambda_{0}, u_{0}, v_{0}\right)\left(\begin{array}{c}
\xi \\
\eta
\end{array}\right)=\left(\begin{array}{c}
-\xi \\
0 \\
0
\end{array}\right)
$$

We can show that $D_{\lambda(u, v)} \mathcal{F}\left(\lambda_{0}, 0, \theta_{\mu}\right)\left(\Phi_{1}, \Phi_{2}\right)^{t} \notin R\left(D_{(u, v)} \mathcal{F}\left(\lambda_{0}, 0, \theta_{\mu}\right)\right)$. Indeed, suppose that there exists $(\xi, \eta) \in X$ such that $D_{(u, v)} \mathcal{F}\left(\lambda_{0}, 0, \theta_{\mu}\right)(\xi, \eta)^{t}=\left(-\Phi_{1}, 0,0\right)$, and so

$$
-\Delta \xi+V^{\prime}(0) \operatorname{div}\left(\xi \nabla \theta_{\mu}\right)-\lambda_{0} \xi=-\Phi_{1} \quad \text { in } \Omega, \quad \partial \xi / \partial n=0 \quad \text { on } \partial \Omega .
$$

Under the change of variable $\xi=e^{V^{\prime}(0) \theta_{\mu}} \varsigma$, the above equation is transformed into

$$
\begin{cases}-\operatorname{div}\left(e^{V^{\prime}(0) \theta_{\mu}} \nabla \varsigma\right)-\lambda_{0} e^{V^{\prime}(0) \theta_{\mu}} \varsigma=-\Phi_{1} & \text { in } \Omega \\ \frac{\partial \varsigma}{\partial n}=0 & \text { on } \Gamma_{1} \\ \frac{\partial \varsigma}{\partial n}+V^{\prime}(0) \mu \frac{\theta_{\mu}}{1+\theta_{\mu}} \varsigma=0 & \text { on } \Gamma_{2}\end{cases}
$$


In a similar way, since $\Phi_{1}$ is an eigenfunction associated to $\Lambda(\mu)$ we can make the change of variable $\Phi_{1}=e^{\alpha \theta_{\mu}} \psi_{1}$, and (3.11) transforms into

$$
\begin{cases}-\operatorname{div}\left(e^{\alpha \theta_{\mu}} \nabla \psi_{1}\right)=\lambda_{0} e^{\alpha \theta_{\mu}} \psi_{1} & \text { in } \Omega, \\ \frac{\partial \psi_{1}}{\partial n}=0 & \text { on } \Gamma_{1}, \\ \frac{\partial \psi_{1}}{\partial n}+V^{\prime}(0) \mu \frac{\theta_{\mu}}{1+\theta_{\mu}} \psi_{1}=0 & \text { on } \Gamma_{2} .\end{cases}
$$

Now, multiplying (3.12) by $\psi_{1}$ and (3.13) by $\varsigma$, and subtracting we get

$$
0=\int_{\Omega} \Phi_{1} \psi_{1}
$$

an absurdum.

It can be showed that $R\left(D_{(u, v)} \mathcal{F}\left(\lambda_{0}, 0, \theta_{\mu}\right)\right)$ has co-dimension 1.

Hence, the point $(\lambda, u, v)=\left(\lambda_{1}(\mu), 0, \theta_{\mu}\right)$ is a bifurcation point from the semi-trivial solution $\left(0, \theta_{\mu}\right)$.

Now, we can apply Theorem 4.1 of [14] and conclude the existence of a continuum $\mathcal{C}^{+} \subset \mathbb{R} \times X_{1} \times X_{2}$ of positive solutions of (3.1) emanating from the point $(\lambda, u, v)=$ $\left(\Lambda(\mu), 0, \theta_{\mu}\right)$ such that:

i) $\mathcal{C}^{+}$is unbounded in $\mathbb{R} \times X_{1} \times X_{2}$; or

ii) there exists $\lambda_{\infty} \in \mathbb{R}$ such that $\left(\lambda_{\infty}, \lambda_{\infty}, 0\right) \in \operatorname{cl}\left(\mathcal{C}^{+}\right)$; or

iii) there exists $\bar{\lambda} \in \mathbb{R}$ such that $(\bar{\lambda}, 0,0) \in \operatorname{cl}\left(\mathcal{C}^{+}\right)$.

Alternative iii) is not possible. Indeed, if a sequence of positive solutions $\left(\lambda_{n}, u_{n}, v_{n}\right) \in$ $\operatorname{cl}\left(\mathcal{C}^{+}\right)$such that $\lambda_{n} \rightarrow \bar{\lambda}$ and $\left(u_{n}, v_{n}\right) \rightarrow(0,0)$ uniformly, then denoting by

$$
V_{n}=\frac{v_{n}}{\left\|v_{n}\right\|_{\infty}}
$$

and using the elliptic regularity, we have that $V_{n} \rightarrow V \geq 0$ and non-trivial in $C^{2}(\bar{\Omega})$ with

$$
-\Delta V+V=0 \quad \text { in } \Omega, \quad \frac{\partial V}{\partial n}=0 \quad \text { on } \Gamma_{1}, \quad \frac{\partial V}{\partial n}=\mu V \quad \text { on } \Gamma_{2},
$$

and so $\mu=\mu_{1}$, a contradiction.

Fixed $\mu>\mu_{1}$, we know by Proposition 3.5 that (3.1) does not possess positive solution if $\lambda \leq 0$ or $\lambda$ is large. Moreover, by Proposition 3.3 it follows that $\mathcal{C}^{+}$is bounded in $X$ uniformly on compact subintervals of $\lambda$. Hence, alternative i) does not occur. Therefore, alternative ii) holds. When this alternative occurs, there exists a sequence $\left(\lambda_{n}, u_{n}, v_{n}\right)$ of solutions of (3.1) such that $\left(\lambda_{n}, u_{n}, v_{n}\right) \rightarrow\left(\lambda_{\infty}, \lambda_{\infty}, 0\right)$. Denoting by

$$
V_{n}=\frac{v_{n}}{\left\|v_{n}\right\|_{\infty}}
$$

we obtain that $V_{n} \rightarrow V$ in $C^{2}(\bar{\Omega})$ with

$$
\left(-\Delta+1+c \lambda_{\infty}\right) V=0 \quad \text { in } \Omega, \quad \partial V / \partial n=0 \quad \text { on } \Gamma_{1}, \quad \partial V / \partial n=\mu V \quad \text { on } \Gamma_{2},
$$

that is, $\mu=\mu_{1}\left(\lambda_{\infty}\right)$. So, we can conclude the existence of a coexistence state for

$$
\lambda \in\left(\min \left\{\left(\Lambda(\mu), \lambda_{\infty}\right)\right\}, \max \left\{\left(\Lambda(\mu), \lambda_{\infty}\right)\right\}\right) .
$$

Observe that if $V^{\prime}(0)=0$ then $\Lambda(\mu)=0$. This completes the proof of the theorem. 


\subsection{Local stability}

We study the local stability of the trivial and semi-trivial solutions.

Proposition 3.8. 1. The trivial solution of (3.1) is l. a. s. if $\lambda<0$ and $\mu<\mu_{1}$ and unstable if $\lambda>0$ or $\mu>\mu_{1}$.

2. Assume that $\lambda>0$. The semi-trivial solution $(\lambda, 0)$ is l. a. s. if $\mu<\mu_{1}(\lambda)$ and unstable if $\mu>\mu_{1}(\lambda)$.

3. Assume that $\mu>\mu_{1}$. The semi-trivial solution $\left(0, \theta_{\mu}\right)$ is l. a. s. if $\lambda<\Lambda(\mu)$ and unstable if $\lambda>\Lambda(\mu)$.

Proof. We prove only the third paragraph of the result, the other ones follow similarly. Observe that the stability of $\left(0, \theta_{\mu}\right)$ is given by the real parts of the eigenvalues for which the following problem admits a solution $(\xi, \eta) \in X \backslash\{(0,0)\}$

$$
\begin{cases}-\Delta \xi+\operatorname{div}\left(V^{\prime}(0) \nabla \theta_{\mu} \xi\right)-\lambda \xi=\sigma \xi & \text { in } \Omega \\ \left(-\Delta+1+c \theta_{\mu}\right) \eta=\sigma \eta & \text { in } \Omega \\ \frac{\partial \eta}{\partial n}=\mu\left(\frac{1}{1+\theta_{\mu}}\right)^{2} \eta & \text { on } \Gamma_{2}\end{cases}
$$

Assume that $\xi \equiv 0$, then for some $j \geq 1$ and we have

$$
\sigma=\lambda_{j}\left(-\Delta+1+c \theta_{\mu} ; N, N-\mu\left(\frac{1}{1+\theta_{\mu}}\right)^{2}\right)>\lambda_{1}\left(-\Delta+1 ; N, N-\mu\left(\frac{1}{1+\theta_{\mu}}\right)^{2}\right)>0 .
$$

Suppose now that $\xi \not \equiv 0$, then from the first equation of (3.14) we get that $\lambda+\sigma$ is a real eigenvalue associated to (3.11). Since $\lambda<\Lambda(\mu)$ it follows that $\sigma>0$.

Assume now that $\lambda>\Lambda(\mu)$. Then,

$$
\sigma_{1}:=\lambda_{1}\left(-\Delta+\operatorname{div}\left(V^{\prime}(0) \nabla \theta_{\mu}\right)-\lambda ; N, N\right)<0 .
$$

Denote by $\xi$ a positive eigenfunction associated to $\sigma_{1}$, that is

$$
-\Delta \xi+\operatorname{div}\left(V^{\prime}(0) \nabla \theta_{\mu} \xi\right)-\lambda \xi=\sigma_{1} \xi \quad \text { in } \Omega, \quad \partial \xi / \partial n=0 \quad \text { on } \partial \Omega .
$$

Since $\sigma_{1}<0$, then

$$
\left.\lambda_{1}\left(-\Delta+1+c \theta_{\mu}-\sigma_{1} ; N, N-\mu\left(\frac{1}{1+\theta_{\mu}}\right)^{2}\right)\right)>0,
$$

and so there exists $\eta$ solution of

$$
\left(-\Delta+1+c \theta_{\mu}\right) \eta=\sigma_{1} \eta \quad \text { in } \Omega, \quad \frac{\partial \eta}{\partial n}=\mu\left(\frac{1}{1+\theta_{\mu}}\right)^{2} \eta \quad \text { on } \Gamma_{2} .
$$

Then, $\sigma_{1}<0$ is an eigenvalue of $(3.14)$ with the eigenfunction associated $(\xi, \eta)$, so $\left(0, \theta_{\mu}\right)$ is unstable. 


\section{Convergence to the semi-trivial solution $(\lambda, 0)$}

Now, we deal with the convergence to the steady-states.

Lemma 4.1. Let $0 \leq \mu<\mu_{1}$ and $\alpha(\mu) \in[0,1]$ the application

$$
\alpha(\mu):=\lambda_{1}(-\Delta+1 ; N, N-\mu) .
$$

Then, there exists $C>0$ such that, for $t>0$, the $v$-solution to (1.1) satisfies

$$
\begin{aligned}
\|v(t)\|_{L^{p}} & \leq C e^{-\beta t}\left\|v_{0}\right\|_{L^{p}} \\
\|v(t)\|_{W^{1, p}} & \leq C t^{-1 / 2} e^{-\beta t}\left\|v_{0}\right\|_{L^{p}} .
\end{aligned}
$$

for all $\beta<\alpha(\mu)$.

Proof. The solutions to the problem

$$
\begin{cases}w_{t}-\Delta w+w=0 & \text { in } \Omega \times\left(0, T_{\max }\right), \\ \frac{\partial w}{\partial n}=0 & \text { on } \Gamma_{1} \times\left(0, T_{\max }\right), \\ \frac{\partial w}{\partial n}=\mu w & \text { on } \Gamma_{2} \times\left(0, T_{\max }\right), \\ w(x, 0)=v_{0}(x) & \text { in } \Omega .\end{cases}
$$

are supersolutions to the $v$-equation of (1.1), therefore $v \leq w$. Now, we define the sectorial operator

$$
A_{p}:-\Delta+I \text {. }
$$

Therefore,

$$
w(x, t)=e^{-t A_{p}} v_{0}
$$

Picking $D\left(A_{p}\right)=W_{\mathcal{B}(\mu)}^{2, p}$, where

$$
\mathcal{B}(\mu)=\left\{\frac{\partial}{\partial n}=0 \text { on } \Gamma_{1}, \quad \frac{\partial}{\partial n}-\mu I=0 \text { on } \Gamma_{2}\right\}
$$

and thanks to [10, Theorem 1.3.4] we have the first assertion. For the second assertion we pick $D\left(A_{p}\right)=H_{\mathcal{B}(\mu)}^{2, p}$, and we apply [3, (7.10)] together with [10, Theorem 1.3.4] (look the proof of Lemma 2.4 and take into account $[3,(5.2)])$.

Our purpose is to show the convergence to the steady states for $u$. To this end we distinguish separately the cases $\lambda=0, \lambda>0$.

\subsection{Case $\lambda=0$.}

Lemma 4.2. Let $\tau, k>0$ and $y \in C(\tau,+\infty) \cap L^{1}(\tau,+\infty), y^{\prime} \in L^{1}(\tau,+\infty)$. If

$$
\lim _{t \rightarrow+\infty} \int_{t}^{t+k}\left(|y(s)|+\left|y^{\prime}(s)\right|\right) d s=0
$$

then $\lim _{t \rightarrow+\infty}|y(t)|=0$. 
Proof. Let us assume that $\lim _{t \rightarrow+\infty}|y(t)| \neq 0$, then there exists a sequence $\left\{t_{n}\right\}_{n \in \mathrm{N}}, t_{n} \rightarrow$ $+\infty$, such that

$$
\left|y\left(t_{n}\right)\right|>C>0, \quad \forall n \geq n_{0} .
$$

We pick $\theta \in(0, k]$, then for all $n \geq n_{0}$ we have

$$
|| y\left(t_{n}+\theta\right)|-| y\left(t_{n}\right)|| \leq\left|y\left(t_{n}+\theta\right)-y\left(t_{n}\right)\right| \leq \int_{t_{n}}^{t_{n}+\theta}\left|y^{\prime}(s)\right| d s \leq \int_{t_{n}}^{t_{n}+k}\left|y^{\prime}(s)\right| d s .
$$

Therefore $|y(s)|>C / 2$ for all $s \in\left[t_{n}, t_{n}+k\right], n \geq n_{0}$. The last assertion contradicts the fact that

$$
\lim _{n \rightarrow+\infty} \int_{t_{n}}^{t_{n}+k}|y(s)| d s=0 .
$$

Theorem 4.3. Assume that $0 \leq \mu<\mu_{1}$ and $\lambda=0$, then

$$
\lim _{t \rightarrow+\infty}\|u(\cdot, t)\|_{C(\bar{\Omega})}=0 .
$$

Proof. After integrating in the space variable the $u$-equation of (1.1) we get

$$
\begin{aligned}
\int_{\Omega} u_{t} & =\int_{\partial \Omega}\left(\frac{\partial u}{\partial n}-V(u) \frac{\partial v}{\partial n}\right)-\int_{\Omega} u^{2} \\
& =-\mu \int_{\Gamma_{2}} \frac{V(u) v}{1+v}-\int_{\Omega} u^{2} .
\end{aligned}
$$

So, integrating the last expression in the time variable between $(\tau, t)$ we obtain

$$
\mu \int_{\tau}^{t} \int_{\Gamma_{2}} \frac{V(u) v}{1+v}+\int_{\tau}^{t} \int_{\Omega} u^{2}=\int_{\Omega} u(\tau)-\int_{\Omega} u(t)
$$

In particular from (4.16) we have

$$
\int_{\tau}^{t} \int_{\Omega} u^{2} \leq\|u(\tau)\|_{L^{1}} \quad \forall t>\tau .
$$

On the other hand, multiplying the $u$-equation of (1.1) by $u$ and integrating in the space variable we obtain

$$
\begin{aligned}
\frac{d}{2 d t} \int_{\Omega} u^{2} & =\int_{\Omega}\left(-|\nabla u|^{2}+V(u) \nabla v \cdot \nabla u-u^{3}\right)-\mu \int_{\Gamma_{2}} \frac{V(u) u v}{1+v} \\
& \leq(\epsilon-1) \int_{\Omega}|\nabla u|^{2}+C(\epsilon) \int_{\Omega}|\nabla v|^{2}-\mu \int_{\Gamma_{2}} \frac{V(u) u v}{1+v}-\int_{\Omega} u^{3}
\end{aligned}
$$

Therefore, we infer

$$
\frac{d}{2 d t} \int_{\Omega} u^{2}+(1-\epsilon) \int_{\Omega}|\nabla u|^{2} \leq C(\epsilon)\|v\|_{W^{1,2}}^{2}
$$

and after integrating in time, thanks to Lemma 4.1 we obtain

$$
\int_{\Omega} u(t)^{2}-\int_{\Omega} u(\tau)+(1-\epsilon) \int_{\tau}^{t} \int_{\Omega}|\nabla u|^{2} \leq C(\epsilon) \int_{\tau}^{t} s^{-1} e^{-2 \beta s}\left\|v_{0}\right\|_{p}
$$


In particular we deduce

$$
\int_{\tau}^{t} \int_{\Omega}|\nabla u|^{2} \leq C \quad \forall t>\tau
$$

Now, using the fact that $\|u(t)\|_{C(\bar{\Omega})} \leq C$ for all $t>0$, we have

$$
\left|\frac{d}{2 d t} \int_{\Omega} u^{2}\right| \leq C \int_{\Omega}|\nabla u|^{2}+C(\epsilon)\|v\|_{W^{1,2}}^{2}+C \mu \int_{\Gamma_{2}} \frac{V(u) v}{1+v}+C \int_{\Omega} u^{2}
$$

Thanks to (4.16), we get

$$
\int_{\tau}^{t}\left|\frac{d}{2 d t} \int_{\Omega} u^{2}\right| \leq C \quad \forall t>\tau
$$

Finally, estimates (4.17) and (4.19) together with Lemma 4.2 entail

$$
\lim _{t \rightarrow+\infty}\|u(\cdot, t)\|_{L^{2}}=0
$$

Also thanks to $\|u(t)\|_{C(\bar{\Omega})} \leq C$ for all $t>0$ we obtain

$$
\lim _{t \rightarrow+\infty}\|u(\cdot, t)\|_{L^{p}}=0, \quad \forall p>2 .
$$

We observe that Lemma 2.9 together with [10, Theorem 1.6.1] assures that

$$
\|u(\cdot, t)\|_{W^{k, p}} \leq C \quad \forall k<1, p \geq 2 .
$$

Next, the Gagliardo-Nirenberg inequality entails

$$
\|u(\cdot, t)\|_{W^{m, p}} \leq C\|u(\cdot, t)\|_{W^{k, p}}^{\theta}\|u(\cdot, t)\|_{L^{p}}^{1-\theta}
$$

for $m<k \theta, \theta \in(0,1)$. Therefore, we have

$$
\lim _{t \rightarrow+\infty}\|u(\cdot, t)\|_{W^{m, p}} \leq C \lim _{t \rightarrow+\infty}\|u(\cdot, t)\|_{L^{p}}^{1-\theta}=0
$$

and the Theorem can be easily concluded picking $m$ such that $m-N / p>0$ thanks to the Sobolev embedding.

\subsection{Case $\lambda>0$.}

In order to do that we impose the following condition. Assume that there exists $t_{0}$ such that

$$
u(\cdot, t)>\delta_{0}>0, \forall t>t_{0}>0 .
$$

Next, we show the long time behavior for $u$ under the hypothesis $(H)$ and after we will give sufficient conditions on $V(u)$ that imply $(H)$.

Theorem 4.4. Let $0 \leq \mu<\mu_{1}$ and assume the the hypothesis $(H)$ is satisfied, then there exists $\theta>0$ such that

$$
\|u(\cdot, t)-\lambda\|_{C(\bar{\Omega})} \leq C e^{-\theta t}
$$

for all $t \geq t_{0}$. 
Proof. On multiplying the $u$-equation by $u-\lambda$ we have

$$
\begin{aligned}
\frac{d}{2 d t} \int_{\Omega}(u-\lambda)^{2} & =-\int_{\Omega}|\nabla u|^{2}+\int_{\Omega} V(u) \nabla v \cdot \nabla u+ \\
& -\mu \int_{\Gamma_{1}} \frac{v}{1+v} V(u)(u-\lambda)-\int_{\Omega} u(u-\lambda)^{2} \\
& \leq-\frac{1}{2} \int_{\Omega}|\nabla u|^{2}+\frac{\|V\|_{L^{\infty}}^{2}}{2} \int_{\Omega}|\nabla v|^{2}+ \\
& +\mu\|V(u)(u-\lambda)\|_{L^{2}\left(\Gamma_{1}\right)}\left(\int_{\Gamma_{1}} \frac{v^{2}}{(1+v)^{2}}\right)^{1 / 2}-\int_{\Omega} u(u-\lambda)^{2} .
\end{aligned}
$$

Having in mind that $(1+v)^{2} \geq 1$, the hypothesis $(H)$ and the Sobolev trace embedding

$$
W^{1,2}(\Omega) \hookrightarrow L^{2}(\partial \Omega)
$$

we get

$$
\frac{d}{d t} \int_{\Omega}(u-\lambda)^{2}+2 \delta_{0} \int_{\Omega}(u-\lambda)^{2} \leq C\|v\|_{W^{1,2}}^{2}+\mu C\|v\|_{W^{1,2}}
$$

Easily, from Lemma 4.1 we can deduce

$$
\|u(\cdot, t)-\lambda\|_{L^{2}}^{2} \leq C e^{-\theta_{1} t}
$$

for $0<\theta_{1}<\min \left\{2 \delta_{0}, \beta\right\}$. At this point we can argue exactly as in the end of Theorem 4.3 to conclude

In the rest of the paper we give conditions on $V$ that imply $(H)$. Such conditions on $V$ involve only the behavior of $V$ around zero. Roughly speaking we require a superlinear grow of $V$ around zero. From now on we assume that there exist $C, \delta_{0}>0, k>1+N / 2$, $j>N / 2$ such that

$$
(H 1) \quad 0<V(s)<C s^{k}, \quad\left|V^{\prime}(s)\right| \leq C s^{j}
$$

for all $s \in\left(0, \delta_{0}\right)$.

Remark 4.5. The condition (H1) is satisfied, for example, for functions $V(u)=\frac{u^{\alpha}}{1+u^{\alpha}}$ with $\alpha>1+N / 2$.

Lemma 4.6. Assume that $0 \leq \mu<\mu_{1}$ and that $(H 1)$ is satisfied then $(H)$ is verified.

Proof. Let $\delta>0$ a constant to be fixed. After multiplying the $u$-equation by $(u-\delta)_{-}$and integrating in the space variable we have

$$
\begin{aligned}
\frac{d}{2 d t} \int_{\Omega}(u-\delta)_{-}^{2} & =-\int_{\Omega}(\nabla u-V(u) \nabla v) \cdot \nabla(u-\delta)_{-}+\int_{\partial \Omega}\left(\frac{\partial u}{\partial n}-V(u) \frac{\partial v}{\partial n}\right)(u-\delta)_{-}+ \\
& +\int_{\Omega} u(\lambda-u)(u-\delta)_{-} \\
& =-\int_{\Omega}\left|\nabla(u-\delta)_{-}\right|^{2}+\int_{\Omega} V(u) \nabla v \cdot \nabla(u-\delta)_{-}- \\
& -\int_{\Gamma_{1}} V(u) \mu \frac{v}{1+v}(u-\delta)_{-}+\int_{\Omega} u(\lambda-u)(u-\delta)_{-} \\
& =-\int_{\Omega}\left|\nabla(u-\delta)_{-}\right|^{2}+\int_{\Omega_{\delta}} V(u) \nabla v \cdot \nabla(u-\delta)_{-}- \\
& -\mu \int_{\Gamma_{\delta}} \frac{v}{1+v} V(u)(u-\delta)_{-}+\int_{\Omega} u(\lambda-u)(u-\delta)_{-},
\end{aligned}
$$


where

$$
\Omega_{\delta}:=\{x \in \Omega: u(x)<\delta\}, \quad \Gamma_{\delta}:=\left\{x \in \Gamma_{1}: u(x)<\delta\right\} .
$$

Next, we apply the Cauchy-Schwartz inequality and we get

$$
\begin{aligned}
\frac{d}{2 d t} \int_{\Omega}(u-\delta)_{-}^{2} & \leq(\epsilon-1) \int_{\Omega}\left|\nabla(u-\delta)_{-}\right|^{2}+C(\epsilon) \int_{\Omega_{\delta}} V^{2}(u)|\nabla v|^{2}- \\
& -\mu \int_{\Gamma_{\delta}} \frac{v}{1+v} V(u)(u-\delta)_{-}+\int_{\Omega} u(\lambda-u)(u-\delta)_{-} \\
& \leq(\epsilon-1) \int_{\Omega}\left|\nabla(u-\delta)_{-}\right|^{2}+C(\epsilon) \sup _{s \in(0, \delta)} V^{2}(s) \int_{\Omega}|\nabla v|^{2}- \\
& -\mu \int_{\Gamma_{\delta}} \frac{v}{1+v} V(u)(u-\delta)_{-}+\int_{\Omega} u(\lambda-u)(u-\delta)_{-}
\end{aligned}
$$

Let

$$
f(\delta):=\sup _{s \in(0, \delta)} V^{2}(s)
$$

After applying the Cauchy-Schwartz inequality to the boundary we obtain

$$
\begin{aligned}
\frac{d}{2 d t} \int_{\Omega}(u-\delta)_{-}^{2} & \leq(\epsilon-1) \int_{\Omega}\left|\nabla(u-\delta)_{-}\right|^{2}+C(\epsilon) f(\delta) \int_{\Omega}|\nabla v|^{2}+\mu \widetilde{\epsilon} \int_{\Gamma_{1}} \frac{v^{2}}{(1+v)^{2}}+ \\
& +\mu C(\widetilde{\epsilon}) \int_{\Gamma_{\delta}} V(u)^{2}(u-\delta)_{-}^{2}+\int_{\Omega} u(\lambda-u)(u-\delta)_{-}
\end{aligned}
$$

Thanks to the Sobolev trace embedding $W^{1,2}(\Omega) \hookrightarrow L^{2}(\partial \Omega)$ and having in mind that $(v+1)^{2} \geq 1$, we have

$$
\begin{gathered}
\int_{\Gamma_{1}} V(u)^{2}(u-\delta)_{-}^{2} \leq C\left(\int_{\Omega} V^{2}(u)(u-\delta)_{-}^{2}+\int_{\Omega}\left(2(u-\delta)_{-}^{2} V^{\prime}(u)^{2}+2 V^{2}(u)\right)\left|\nabla(u-\delta)_{-}\right|^{2}\right), \\
\mu \widetilde{\epsilon} \int_{\Gamma_{1}} \frac{v^{2}}{(1+v)^{2}} \leq C \widetilde{\epsilon}\|v\|_{W^{1,2}}^{2}
\end{gathered}
$$

Therefore, we obtain

$$
\begin{aligned}
\frac{d}{2 d t} \int_{\Omega}(u-\delta)_{-}^{2} & \leq(\epsilon-1) \int_{\Omega}\left|\nabla(u-\delta)_{-}\right|^{2}+C(\epsilon) f(\delta) \int_{\Omega}|\nabla v|^{2}+C \widetilde{\epsilon}\|v\|_{W^{1,2}}^{2}+ \\
& +C(\widetilde{\epsilon}) \int_{\Omega} V^{2}(u)(u-\delta)_{-}^{2}+C(\widetilde{\epsilon}) \int_{\Omega}\left(2(u-\delta)_{-}^{2} V^{\prime}(u)^{2}+2 V^{2}(u)\right)\left|\nabla(u-\delta)_{-}\right|^{2}+ \\
& +\int_{\Omega} u(\lambda-u)(u-\delta)_{-} .
\end{aligned}
$$

Let

$$
g(\delta):=\sup _{s \in(0, \delta)}\left(2(s-\delta)_{-}^{2} V^{\prime}(s)^{2}+2 V^{2}(s)\right)
$$

Hence, taking into account that $-\delta<(u-\delta)_{-}$, we get

$$
\begin{aligned}
\frac{d}{2 d t} \int_{\Omega}(u-\delta)_{-}^{2} & \leq(\epsilon+C(\widetilde{\epsilon}) g(\delta)-1) \int_{\Omega}\left|\nabla(u-\delta)_{-}\right|^{2}+(C(\epsilon) f(\delta)+\mu \widetilde{\epsilon})\|v\|_{W^{1,2}}^{2}+ \\
& +\int_{\Omega} u(u-\delta)_{-}\left(\lambda-u-C\left(\widetilde{\epsilon} \frac{V^{2}(u)}{u} \delta\right)\right.
\end{aligned}
$$


Observe that if

$$
C(\widetilde{\epsilon}) g(\delta)<1-\epsilon
$$

and

$$
C(\widetilde{\epsilon}) \frac{V^{2}(s)}{s} \delta \leq \lambda-\delta
$$

then, thanks to the strong maximum principle for the $u$-equation and Lemma 4.1 we have that

$$
\left\|(u-\delta)_{-}(t)\right\|_{2}^{2} \leq(2 C(\epsilon) f(\delta)+2 \mu \widetilde{\epsilon}) C(\beta)
$$

We try to prove that

$$
\left\|(u-\delta)_{-}\right\|_{2}^{2} \leq \frac{\delta^{2 \alpha}}{2 C_{p}}
$$

for any $\alpha>1$ and $C_{p}>1$ is a constant to be given. To this end, we impose the following conditions

$$
2 C(\epsilon) f(\delta) C(\beta) \leq \frac{\delta^{2 \alpha}}{4 C_{p}}
$$

and

$$
\mu \widetilde{\epsilon} C(\beta) \leq \frac{\delta^{2 \alpha}}{4 C_{p}}
$$

We have to check that there exist $\epsilon, \widetilde{\epsilon}, \delta>0$ such that conditions (4.27)-(4.30) are satisfied simultaneously. Let us observe that the inequality (4.30) is satisfied for

$$
\widetilde{\epsilon} \leq \frac{\delta^{2 \alpha}}{4 C_{p} \mu C(\beta)},
$$

so,

$$
C(\widetilde{\epsilon})=C(\beta, \mu) \delta^{-2 \alpha} .
$$

Then, we pick $\epsilon=1 / 2$, thus $C(\epsilon)=1 / 2$. Thanks to (H1), we have

$$
f(\delta) C(\beta)=\sup _{\delta \in(0, \delta)} V^{2}(s) C(\beta) \leq C \delta^{2 k} C(\beta) .
$$

Hence, for $\alpha<k$ and $\delta$ sufficiently small the inequality (4.29) is satisfied . Now, owing to $(H 1)$, we observe that

$$
\begin{aligned}
C(\widetilde{\epsilon}) \frac{V^{2}(s)}{s} \delta & \leq C(\widetilde{\epsilon}) \sup _{s \in(0, \delta)} \frac{V^{2}(s)}{s} \delta \\
& \leq C(\widetilde{\epsilon}) \delta^{2 k}
\end{aligned}
$$

So, condition (4.28) can be assured for $\alpha<k$ and $\delta$ small enough. Now, it is straightforward to see that condition (4.27) it is also satisfied for $1<\alpha<\min \{k, 1+j\}$. Next we use interpolation between $L^{p}$ spaces to obtain

$$
\begin{aligned}
\left\|(u-\delta)_{-}\right\|_{2 / \theta_{1}} & \leq\left\|(u-\delta)_{-}\right\|_{2}^{\theta_{1}}\left\|(u-\delta)_{-}\right\|_{\infty}^{1-\theta_{1}} \\
& \leq \delta^{\alpha \theta_{1}} \delta^{1-\theta_{1}}=\delta^{1+(\alpha-1) \theta_{1}}
\end{aligned}
$$

Next, we apply [3, Theorem 7.2] for $s_{0}=0$ and $s_{1}=1$. So, we infer

$$
\left\|(u-\delta)_{-}\right\|_{W^{\theta, 2 / \theta_{1}}} \leq C\left\|(u-\delta)_{-}\right\|_{W^{1,2 / \theta_{1}}}^{\theta}\left\|(u-\delta)_{-}\right\|_{L^{2 / \theta_{1}}}^{1-\theta},
$$


In order to have $W^{\theta, 2 / \theta_{1}} \hookrightarrow L^{\infty}$ we pick $\theta_{1}$ such that

$$
\theta-\frac{N \theta_{1}}{2}>0
$$

Thus, $\theta>\frac{N \theta_{1}}{2}$. It should be satisfied also that

$$
(1-\theta)\left(1+(\alpha-1) \theta_{1}\right)>1
$$

So,

$$
1<\left(1-\frac{N \theta_{1}}{2}\right)\left(1+(\alpha-1) \theta_{1}\right)
$$

After some algebra, it is possible to find $\theta, \theta_{1} \in(0,1)$ satisfying (4.31) and (4.32) if and only if $\alpha>1+\frac{N}{2}$. Therefore using the uniform bound in time in $W^{1, p}(\Omega)$ (that is a consequence of [3, Theorem 15.5]), the Sobolev embedding and picking $C_{p}$ properly we obtain

$$
\left\|(u-\delta)_{-}(t)\right\|_{\infty} \leq \frac{\delta}{2},
$$

for $t \geq t_{0}>0$. The last estimate concludes easily the Lemma.

\section{Interpretation}

In this paper we have analyzed a problem modelling the angiogenesis. For that, we have included a nonlinear chemotactic sensitivity, a logistic term to model the growth rate of the CEs and a nonlinear term at the boundary of the tumor. We have shown the validity of the model proving the existence and uniqueness of positive solution of the model.

Let us interpret some of our results. Fix the growth rate of CEs, that is, fix $\lambda>0$. Then, we can define $\mu_{2}(\lambda)$ as

$$
\mu_{2}(\lambda):=\max \{\mu: \lambda=\Lambda(\mu)\} .
$$

It is clear that $\mu_{2}(\lambda)=+\infty$ if $V^{\prime}(0)=0$.

With this notation, we know that for $\mu \in\left(\mu_{1}(\lambda), \mu_{2}(\lambda)\right)$ there exists a coexistence state, and so the angiogenesis occurs. However, in the case $V^{\prime}(0)>0$, for $\mu$ large there does not exist coexistence state and $\left(0, \theta_{\mu}\right)$ is stable, that is, we can avoid the angiogenesis, i.e., if the tumor generates a lot of TAF, this competes with the CEs and CEs death. However, this does not occur when $V^{\prime}(0)=0$, due to the fact that in this case the chemotactic sensitivity is too small and then CEs does not move quickly and they do not come into contact with TAF.

Acknowledgements. Supported by MEC under grants MTM2006-07932.

\section{References}

[1] R. Adams. Sobolev spaces, Academic Press, New York, (1975). 
[2] S. Agmon, A. Douglis and L. Nirenberg, Estimates near the boundary for solutions of elliptic partial differential equations satisfying general boundary conditions, I. Comm. Pure Appl. Math., 12 (1959) 623-727.

[3] H. Amann, Nonhomogeneous Linear and Quasilinear Elliptic and Parabolic Boundary Value Problems, Eds. Schmeisser and Triebel, Function Spaces, Differential Operators and Nonlinear Analysis, Teubner Texte zur Mathematik, 133 (1993) 9-126.

[4] H. Amann, Nonlinear elliptic equations with nonlinear boundary conditions, In: New Developments in differential equations (Eckhaus, W. ed.), Math Studies, 21, NorthHolland, Amsterdam, (1976), 43-63.

[5] S. Cano-Casanova and J. López-Gómez, Properties of the principal eigenvalues of a general class of non-classical mixed boundary value problems, J. Differential Equations 178 (2002), 123-211.

[6] M. G. Crandall and P. H. Rabinowitz, Bifurcation from simple eigenvalues, J. Funct. Anal., 8, 321-340 (1971).

[7] M. Delgado and A. Suárez, Study of an elliptic system arising from angiogenesis with chemotaxis and flux at the boundary, JDE (2008).

[8] J. García-Melián, J. D. Rossi and J. Sabina de Lis, Existence and uniqueness of positive solutions to elliptic problems with nonlinear mixed boundary conditions, preprint.

[9] D. Gilbarg and N. S. Trudinger, Elliptic partial differential equations of second order, Springer Verlag (1983).

[10] D. Henry, Geometric theory of semilinear parabolic equations, Lecure Notes Math. 840, Springer 1981.

[11] T. Hillen and K. J. Painter, A user's guide to PDE models for chemotaxis, J. Math. Biol., (2008).

[12] T. Hillen and K. J. Painter, Global existence for a parabolic chemotaxis model with preventing of overcrowding, Adv. Appl. Math., 26 (2001), 280-301.

[13] D. Horstmann and M. Winkler, Boundedness vs Blow-up in a chemotaxis sytem, J. Differential Equations 215 (2005), 52-107.

[14] J. López-Gómez, Nonlinear eigenvalues and global bifurcation: Application to the search of positive solutions for general Lotka-Volterra reaction-diffusion systems with two species, Diff. Int. Eqns., 7 (1994), 1427-1452.

[15] N. V. Mantzaris, S. Webb and H. G. Othmer, Mathematical modeling of tumorinduced angiogenesis, J. Math. Biol. 49 (2004), 111-187.

[16] K. J. Painter and T. Hillen, Volume-filling and quorum-sensing in models for chemosensitive movement, Can. Appl. Math. Quart., 10 (2002), 501-543.

[17] C. V. Pao, Nonlinear parabolic and elliptic equations, Plenum, New York, 1992. 
[18] L. TARTAR. An introduction to Sobolev spaces and interpolation spaces, Lecture Notes of the Unione Matematica Italiana, 3, Springer, Berlin, (2007).

[19] K. Umezu, Nonlinear elliptic boundary value problems suggested by fermentation, NoDEA Nonlinear Differential Equations Appl., 7 (2000), 143-155.

[20] C. Walker and G. F. WebB, Global existence of classical solutions for a haptotaxis model, SIAM J. Math. Anal. 38 (2007), 1694-1713. 